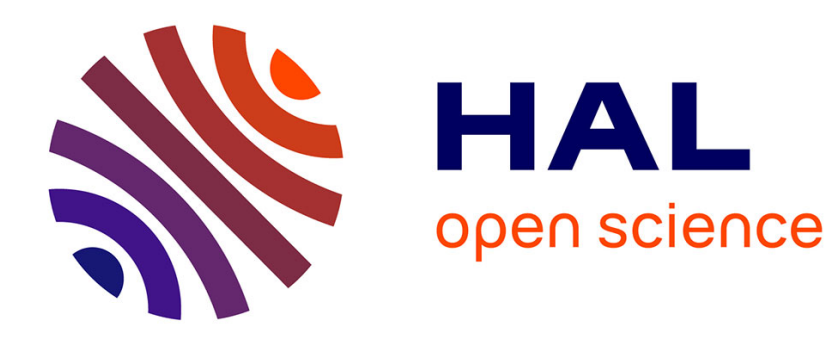

\title{
Splitting Risks in Insurance Markets With Adverse Selection
}

Pierre Picard

\section{To cite this version:}

Pierre Picard. Splitting Risks in Insurance Markets With Adverse Selection. 2017. hal-01448322v4

\section{HAL Id: hal-01448322}

\section{https://hal-polytechnique.archives-ouvertes.fr/hal-01448322v4}

Preprint submitted on 8 Sep 2017

HAL is a multi-disciplinary open access archive for the deposit and dissemination of scientific research documents, whether they are published or not. The documents may come from teaching and research institutions in France or abroad, or from public or private research centers.
L'archive ouverte pluridisciplinaire HAL, est destinée au dépôt et à la diffusion de documents scientifiques de niveau recherche, publiés ou non, émanant des établissements d'enseignement et de recherche français ou étrangers, des laboratoires publics ou privés. 


\section{ECOLE POLYTECHNIQUE}

\section{Splitting Risks in Insurance Markets With Adverse Selection}

Pierre PICARD

August 24, 2017

Cahier $\mathrm{n}^{\circ}$ 2017-01

\section{DEPARTEMENT D'ECONOMIE}

Route de Saclay

91128 PALAISEAU CEDEX

(33) 169333410

http://www.portail.polytechnique.edu/economie/fr

weronika-natalia.leduc@polytechnique.edu 


\title{
Splitting Risks in Insurance Markets with Adverse Selection
}

\author{
Pierre Picard *
}

August 24st, 2017

\begin{abstract}
We characterize the design of insurance schemes when policyholders face several insurable risks in a context of adverse selection. Splitting risks emerges as a feature of second-best Pareto-optimal allocations. This may take the form of risk-specific contracts, or of contracts where risks are bundled but subject to differential coverage rules - such as risk-specific copayments -, combined with a deductible, an out-ofpocket maximum or a cap on coverage.
\end{abstract}

${ }^{*}$ CREST, Ecole Polytechnique, 91128, Palaiseau Cedex, France. Email: pierre.picard@polytechnique.edu 


\section{Introduction}

Most people are simultaneously affected by several insurable risks, including property, casualty, liability and health risks. It is a fact that these various categories of risks may require specific underwriting and claim-handling skills that are not uniformly shared by all insurers. This is a sufficient reason why we may cover different risks through different insurers, and thus, for instance, why many people purchase automobile and medical insurance from different insurers.

Understanding why similar risks incurred by the same economic unit (e.g., a family) are not covered by the same insurance contract is less obvious. To take a concrete example, why is it the case that, most of the time, the automobile risks of both members of a couple who drive different cars are covered by separate contracts, with specific premium and deductibles, and similarly, why the health care risks of these two people may be covered by independent contracts? ${ }^{1}$

These very simple questions do not have simple answers. When there are no transaction costs, risk averse individuals would optimally purchase full coverage with actuarially fair insurance premium, and, obviously, in such a case it does not matter whether the risks of both members of a couple are covered through specific contracts or through a single contract. This is no longer true when insurers charge loaded premiums and households react by purchasing partial coverage. A variant of the wellknown "theorem of the deductible" says that, under proportional loading, households should be covered by an "umbrella policy" with full marginal coverage of the total losses beyond an aggregate deductible. ${ }^{2}$ The fact that they may choose to be

\footnotetext{
${ }^{1}$ These questions are legitimate insofar as both members of the couples have identical access rights to the same set of contracts, and if they jointly manage their budget. Matters should be seen differently in a collective approach to family decision making, where a household is described as a group of individuals with their own preferences, and among whom there is a coperative or non-cooperative decision process; see Bourguignon and Chiappori (1992).

${ }^{2}$ Umbrella insurance usually refers to coverage provided as a complement to other policies, particularly automobile and homeowners' liability policies. Like others before us, we use this terminology because the principle of umbrella policies is to globally protect policyholders against uncovered risks, without reference to the specificity of these risks. Following Eeckhoudt et al. (1991), who considered the particular case of a binomial distribution, more generally, Gollier and Schlesinger (1995) established the optimality of an umbrella policy under linear transaction costs. As shown by Gollier (2013), this property
} 
covered by separate insurance policies is inconsistent with this conclusion.

A similar question arises with respect to the coverage of various risks incurred by an individual. This is particularly the case in medical insurance. In an umbrella policy approach, the insurance indemnity depends on total medical expenses measured on a per period basis. However, much more frequently, various medical services (e.g. visits to general practitioners, visits to specialists, inpatient care, dental care, etc) are bundled in a single contract, but the insurance indemnity depends on service specific copayments, coinsurance rates or policy limits, frequently combined with an aggregate deductible, an out-of-pocket maximum or a cap on the indemnity. Here also, this casual observation of medical insurance contracts is at odds with the optimality of an umbrella policy. Likewise, homeowner insurance usually provides bundled coverage for both property and liability risks, with different deductibles and policy limits for these risks.

The fact that risk-specific policies and differential coverage of risks bundled in a contract are so frequent suggests that splitting risks is often an optimal answer to the insurance choice problem. Umbrella policies may be suboptimal for various reasons that are ignored in the standard model of insurance demand, and that would be worth further exploring. Most of them are probably related to asymmetries of information between insurer and insured, for instance when the two members of a couple do not react to financial incentives in the same way under moral hazard, or when medical expenses can be more or less easily monitored by insurers, depending on the type of health care service (e.g. visit to general practionners vs inpatient care). ${ }^{3}$

In this paper, we will analyze how the splitting of risks is a rational answer to the adverse selection problem. We will consider a setting where households face several risk exposures (only two, for the sake of simplicity), with hidden information about the type of each of them: they may be high risk or low risk. For instance, in the case of health insurance the two risk

as well as the optimality of a straight deductible contract hold when policyholders dislike any zero-mean lottery that would be added to their final wealth, which is more general than the case of risk aversion under the expected utility criterion.

${ }^{3}$ Cohen (2006) considers a related issue. She analyzes the determinants of the deductible in insurance contracts that cover a risk that may materialize more than once during the life of the policy. She shows that aggregate deductibles may produce higher verification costs and moral hazard costs than per-loss deductibles. In a similar perspective, Li. et al. (2007) analyze the case of automobile insurance in Taiwan, and provide evidence of the incentive effects of increasing per-claim deductibles on the policyholders' behavior. 
exposures may correspond to the medical expenses of each member of a couple, or to different types of medical services. We will have high-risk households with two high-risk exposures, medium-risk households with a high risk and a low risk (with two subgroups according to the identity of the high- and low-risk exposures), and low-risk households with two low risks. An umbrella policy provides coverage for the aggregate losses that may result from the two risk exposures, while a risk-specific policy only covers the loss of a particular risk. In this setting, the coverage of risks may be split by purchasing insurance through two risk-specific policies, such as two automobile insurance policies for the two cars owned by the household, or separate health insurance contracts for each member of a couple. Bearing in mind the case of health insurance, we may also consider that policyholders are individuals who face two additive risks, each of them corresponding to a specific service, such as visits to general practitioners and dental care. In this case, splitting risks refers to the bundling of insurance coverage with service-specific coverage, possibly combined with a deductible, an out-ofpocket maximum or a cap on indemnity.

Crocker and Snow (2011) study a related but different problem. They analyze how the bundling of distinct perils (seen as factors that may be at the origin of a loss) in a single contract with differential deductibles improves the efficiency of insurance markets under adverse selection. They consider a setting with two types of policyholder (high risk and low risk) where a loss may result from various perils, and they show that bundling the coverage of these perils in a single contract facilitates the separation of high risks and low risks. If the probabilities that the loss results from different perils (conditionnally on a loss occurring) differ between high-risk and low-risk individuals, then bundling perils with differential deductibles allows insurers to implement a multidimensional screening that yields a Pareto efficiency gain and makes the existence of a Rothschild-Stiglitz equilibrium more likely.

Our perspective is different. We consider a setting in which each policyholder faces two risks that may be high or low, and potentially one loss per risk. We thus have four types of policyholder and 0,1 or 2 losses. The issue we consider is whether the aggregate loss that may result from multiple risk exposures should be covered through an umbrella policy, and if not, whether risk splitting takes the form of risk specific policies where risk exposures are unbundled, or of a single policy where risks are bundled but different indemnity rules apply for each risk.

We will not try here to analyze the market equilibrium that may arise 
when there is adverse selection about multiple insurable risks, although this should be an ultimate research objective. Our objective will be more modestly limited to the characterization of efficient allocations. Efficiency will be in a second-best sense, meaning that allocations should satisfy the incentive compatibility conditions inherent in a hidden information setting. Implicitly, we presume that either competition forces or market regulation lead to such a second-best Pareto optimal allocation. Our analysis will be carried out within the framework of a model that belongs to the class of multidimensional screening models surveyed by Armstrong and Rochet (1999) and Rochet and Stole (2003). As in these models, technical difficulties arise from the fact that no single crossing assumption exists in such models, and thus that - contrary to what occurs in the standard Rothschild-Stiglitz (1976) setting with a single risk exposure, extended to an arbitrary number of types by Spence (1978) - the state-contingent wealth allocated to a household may be jointly affected by the incentive constraints of several other households with different types of risk exposure.

The main lesson learned from the characterization of efficient allocations will be that risk splitting facilitates the separation of risk types. The intuition for this result is as follows. As in the Rothschild-Stiglitz (1976) model, unsurprisingly, at any second-best Pareto-optimal allocation high-risk households are fully covered, which may correspond to an umbrella policy or to two risk-specific policies. They are deterred from choosing the risk coverage of the "upward adjacent" type of household - which is the medium risk in the present model - by providing partial coverage to this type. In our multiple risk setting, partial coverage may correspond to several insurance schemes, such as, for instance, a uniform proportional coinsurance for the two risk exposures. It turns out that full coverage for the high-risk exposure and partial coverage for the low-risk exposure is the most efficient way to separate highand medium-risk households, since the existence or the absence of such a low risk exposure is the only difference between these two categories of household. This requires that medium-risk households be covered by risk-specific insurance policies, with full coverage for their high risk and partial coverage or even no coverage for their low risk.

The situation is different for low-risk households, since their two risk exposures are homogeneous (both are low risks), and their insurance policy, to facilitate the separation from medium-risk households, should include decreasing coverage at the margin for both risk exposures. Risk specific policies are not optimal for low-risk households, but splitting the coverage of risk ex- 
posures is again optimal because of the (possibly uneven) pressure of the two types of medium-risk policyholders. It takes a form that will sound very familiar in a health economics perspective: risk-specific contributions of the policyholder in the case of a loss (i.e., in the terminology of health insurance, risk-specific copayments) should be combined with an aggregate deductible, an out-of-pocket maximum or a cap on the insurance indemnity.

We will also consider the case when actuarial premiums are loaded up because of transaction costs. This extension is of particular interest because of the theorem of the deductible that states the optimality of an aggregate deductible when there is proportional loading and no asymmetry of information. Taking into account insurance loading will modify our conclusions about the optimality of full coverage for high-risk households and of riskspecific insurance contracts for medium-risk households, but splitting risks will remain an optimal answer to the adverse selection problem. In particular, high-risk households should be protected by a straight deductible contract, while medium- and low-risk households should be covered by a policy that includes risk-specific copayments combined with an aggregate deductible or an out-of-pocket maximum.

The rest of the paper is arranged as follows. Section 2 presents our model of an insurance market with multiple risks and hidden information about risk types. Section 3 shows why and how risk splitting may be an answer to adverse selection. Section 4 analyzes the effect of transaction costs on insurance schemes. Section 5 concludes. The proofs are in an appendix.

\section{Model}

\subsection{Notations}

We consider an economy where households are affected by two additive financial risk exposures (more briefly, risks), indexed by $r \in\{1,2\}$ and identified by observable characteristics. ${ }^{4}$ We can think of the health care expenses incurred by both members of a couple, hence the "household" terminology. The model may also be interpreted as the case of a single person who faces

\footnotetext{
${ }^{4}$ Hence, households cannot present their risk exposure 1 as belonging to category 2, and vice versa. The analysis would be different if both risk exposures could be substituted, as for instance in the case of property owners seeking fire insurance for a group of two buildings, without special features making it possible to distinguish between them.
} 
two risks, such as doctors' visits and inpatient care in the case of health insurance, or accident and theft risk in the case of automobile risk. For the sake of notational simplicity, we assume that each risk $r$ may lead to the same loss $L$, with probability $p_{\ell}$ or $p_{h}$, with $0<p_{\ell}<p_{h}<1$, and that the occurrence of these losses are independent events. Hence, $r$ is a high risk when the corresponding probability of loss is $p_{h}$ and it is a low risk when the probability is $p_{\ell}$. Thus, there are four household types characterized by a double index $i j$, with $i, j \in\{h, \ell\}$, where $i$ and $j$ are the types of risk exposures $r=1$ and 2, respectively. Types $h h$ and $\ell \ell$ are high-risk and low-risk households because their two risks are either high or low. Types $h \ell$ and $\ell h$ are medium risk households, because one of their risk exposures is high and the other is low. We denote $\lambda_{i j}$ the proportions of type $i j$ households with $\lambda_{h h}+\lambda_{h \ell}+\lambda_{\ell h}+\lambda_{\ell \ell}=1$. Households have private information about their type and all of them have the same initial wealth $w_{0}$.

The state-contingent final wealth of a household is written as $w=\left(w^{00}, w^{01}\right.$, $\left.w^{10}, w^{11}\right)$, where $w^{x y}$ denotes its final wealth in state $(x, y) \in\{0,1\}^{2}$, where $x=0$ when risk $r=1$ does not lead to a loss and $x=1$ when it does, with a similar interpretation of index $y$ for risk $r=2$. For instance, $w^{10}$ is the household's final wealth when only risk 1 is at the origin of a loss.

Households are decision units: they are risk averse and they maximize the expected utility of their aggregate final wealth $w_{f}$, with utility function $u\left(w_{f}\right)$, such that $u^{\prime}>0, u^{\prime \prime}<0$. To obtain more specific results, we may also assume that households are prudent, which corresponds to the additional assumption $u^{\prime \prime \prime}>0$, but we will mention it explicitly when this assumption is needed. The expected utility of a type $i j$ household is denoted $U_{i j}(w)$ for all $i, j \in\{h, \ell\}$, with

$$
\begin{aligned}
U_{h h}(w) \equiv & \left(1-p_{h}\right)^{2} u\left(w^{00}\right)+p_{h}\left(1-p_{h}\right)\left[u\left(w^{01}\right)+u\left(w^{10}\right)\right]+p_{h}^{2} u\left(w^{11}\right), \\
U_{h \ell}(w) \equiv & \left(1-p_{h}\right)\left(1-p_{\ell}\right)\left(w^{00}\right)+p_{\ell}\left(1-p_{h}\right) u\left(w^{01}\right) \\
& +p_{h}\left(1-p_{\ell}\right) u\left(w^{10}\right)+p_{h} p_{\ell} u\left(w^{11}\right) \\
U_{\ell h}(w) \equiv & \left(1-p_{h}\right)\left(1-p_{\ell}\right)\left(w^{00}\right)+p_{h}\left(1-p_{\ell}\right) u\left(w^{01}\right) \\
& +p_{\ell}\left(1-p_{h}\right) u\left(w^{10}\right)+p_{h} p_{\ell} u\left(w^{11}\right) \\
U_{\ell \ell}(w) \equiv & \left(1-p_{\ell}\right)^{2} u\left(w^{00}\right)+p_{\ell}\left(1-p_{\ell}\right)\left[u\left(w^{01}\right)+u\left(w^{10}\right)\right]+p_{\ell \ell}^{2} u\left(w^{11}\right) .
\end{aligned}
$$

The state-contingent final wealth of a type $i j$ household is denoted by $w_{i j}=\left(w_{i j}^{00}, w_{i j}^{01}, w_{i j}^{10}, w_{i j}^{11}\right)$, with expected utility $U_{i j}\left(w_{i j}\right)$. An allocation $\mathcal{A}=$ $\left\{w_{h h}, w_{h \ell}, w_{\ell h}, w_{\ell \ell}\right\}$ specificies the state-contingent final wealth for each type of household. 
Insurance schemes ${ }^{5}$ are characterized by the non-negative indemnity $T^{x y}$ paid by the insurer to the household when there are $x$ and $y$ losses, in risk exposures $r=1$ and 2, respectively, with $(x, y) \in\{0,1\}^{2}$ and $T^{00}=0$, and by the premium $P$ paid in all states by the policyholder to the insurer. If type $i j$ households are protected by an insurance scheme with coverage schedule $T_{i j} \equiv\left(T_{i j}^{01}, T_{i j}^{10}, T_{i j}^{11}\right)$ and premium $P_{i j}$, then we have

$$
w_{i j}^{x y}=w-(x+y) L-P_{i j}+T_{i j}^{x y} \text { for all } x, y \in\{0,1\},
$$

with $T_{i j}^{00}=0$.

In what follows, for the sake of simplicity and realism, we will restrict attention to insurance schemes where the indemnity is comonotone with losses and does not increase more than losses, and thus we assume ${ }^{6}$

$$
\begin{aligned}
& 0 \leq T_{i j}^{x 1}-T_{i j}^{x 0} \leq L \text { if } x \in\{0,1\}, \\
& 0 \leq T_{i j}^{1 y}-T_{i j}^{0 y} \leq L \text { if } y \in\{0,1\},
\end{aligned}
$$

or, equivalently

$$
\begin{aligned}
& L \leq w_{i j}^{x 0}-w_{i j}^{x 1} \leq 0 \text { if } x \in\{0,1\}, \\
& L \leq w_{i j}^{0 y}-w_{i j}^{1 y} \leq 0 \text { if } y \in\{0,1\},
\end{aligned}
$$

for all $i, j \in\{h, \ell\}$.

\section{$2.2 \quad$ Typical multirisk insurance schemes}

Risk-specific policies and umbrella policies are particular forms of insurance schemes that restrict the set of feasible allocations. A risk-specific insurance policy provides coverage for a particular risk. A policy that covers risk 1 is defined by an indemnity $I^{1}$ paid to the household, should risk 1 lead to a loss, and by an insurance premium $Q^{1}$, with similar notations $I^{2}, Q^{2}$ for a

\footnotetext{
${ }^{5}$ We use the terminology "insurance scheme" instead of "insurance policy" or "contract" because, in what follows, an insurance scheme may correspond to the combination of two risk specific insurance policies.

${ }^{6}$ In addition to the fact that overinsurance may be prohibited by law, policyholders would be incentivized to deliberately create damage (e.g. by commiting arson in the case of fire insurance) if coverage were larger than losses.
} 
policy that covers risk 2 . Hence, if type $i j$ households are covered by two risk-specific policies $\left(I_{i j}^{1}, Q_{i j}^{1}\right)$ and $\left(I_{i j}^{2}, Q_{i j}^{2}\right)$, then we have

$$
\begin{aligned}
& T_{i j}^{10}=I_{i j}^{1}, \\
& T_{i j}^{01}=I_{i j}^{2}, \\
& T_{i j}^{11}=I_{i j}^{1}+I_{i j}^{2}, \\
& P_{i j}=Q_{i j}^{1}+Q_{i j}^{2},
\end{aligned}
$$

which induces an allocation that satisfies

$$
w_{i j}^{11}=w_{i j}^{01}+w_{i j}^{10}-w_{i j}^{00} \text { for all } i, j \in\{h, \ell\} .
$$

This characterization can be reversed: any allocation that satisfies (2) is induced by an insurance scheme where type $i j$ households are covered by two type-dependent risk-specific policies.

Under an umbrella policy, the coverage is a function of the household's total loss, and the policy may be written as $\left(J^{1}, J^{2}, P\right)$, where $J^{1}$ and $J^{2}$ denote the indemnity in the case of one or two losses, respectively, and, as before, $P$ is the total premium paid for the coverage of the two risks. Thus, when type $i j$ households are covered by an umbrella policy $\left(J_{i j}^{1}, J_{i j}^{2}, P_{i j}\right)$,we have

$$
\begin{aligned}
& T_{i j}^{10}=T_{i j}^{01}=J_{i j}^{1}, \\
& T_{i j}^{11}=J_{i j}^{2} .
\end{aligned}
$$

The corresponding allocation satisfies

$$
w_{i j}^{01}=w_{i j}^{10} \text { for all } i, j \in\{h, \ell\},
$$

with, here also, an inverse relationship: any allocation that satisfies (3) is induced by an umbrella policy for type $i j$ households.

If an umbrella policy $\left(J_{i j}^{1}, J_{i j}^{2}, P_{i j}\right)$ provides the same state contingent wealth $w_{i j}=\left(w_{i j}^{00}, w_{i j}^{01}, w_{i j}^{10}, w_{i j}^{11}\right)$ as two risk-specific policies $\left(I_{i j}^{1}, Q_{i j}^{1}\right)$ and $\left(I_{i j}^{2}, Q_{i j}^{2}\right)$, then we have

$$
w_{i j}^{11}-w_{i j}^{01}=w_{i j}^{10}-w_{i j}^{00}=w_{i j}^{01}-w_{i j}^{00}
$$

from (2) and (3), and thus

$$
J_{i j}^{2}=2 J_{i j}^{1}
$$


Hence, the indemnity is doubled when the loss is doubled, which means that linear coinsurance is an umbrella policy equivalent to two separate riskspecific policies, both of them providing linear coinsurance at the same rate.

However, risk-specific policies and umbrella policies are far from being the only forms of multirisk insurance schemes. Consider the case of risk-specific copays $c_{i j}^{1}$ and $c_{i j}^{2}$ combined with a deductible $D_{i j}$, and assume $c_{i j}^{1}+D_{i j}<L$ and $c_{i j}^{2}+D_{i j}<L .^{7}$ This gives

$$
\begin{aligned}
& T_{i j}^{10}=L-c_{i j}^{1}-D_{i j}, \\
& T_{i j}^{01}=L-c_{i j}^{2}-D_{i j}, \\
& T_{i j}^{11}=2 L-c_{i j}^{1}-c_{i j}^{2}-D_{i j},
\end{aligned}
$$

Using (1) yields

$$
\begin{aligned}
& c_{i j}^{1}=w_{i j}^{01}-w_{i j}^{11}, \\
& c_{i j}^{2}=w_{i j}^{10}-w_{i j}^{11}, \\
& D_{i j}=w_{i j}^{00}+w_{i j}^{11}-\left(w_{i j}^{10}+w_{i j}^{01}\right) .
\end{aligned}
$$

with $D_{i j} \geq 0$ if

$$
w_{i j}^{00}+w_{i j}^{11} \geq w_{i j}^{10}+w_{i j}^{01},
$$

and we have $T_{i j}^{01}, T_{i j}^{10}, T_{i j}^{11}>0$ if $w_{i j}^{00}-w_{i j}^{01}<L$ and $w_{i j}^{00}-w_{i j}^{10}<L .{ }^{8}$ Conversely, under condition (4), $w_{i j}$ can be sustained by non-negative copays $c_{i j}^{1}, c_{i j}^{2}$ and deductible $D_{i j}$. This equivalence between condition (4) and the sustainability of $w_{i j}$ through copays and a deductible also holds when $T_{i j}^{01}, T_{i j}^{10}$ and/or $T_{i j}^{11}=0$, with $w_{i j}^{00}-w_{i j}^{01}=L, w_{i j}^{00}-w_{i j}^{10}=L$ and/or $w_{i j}^{00}-w_{i j}^{11}=2 L$, respectively. ${ }^{9}$ Thus, more generally, an allocation that satisfies condition (4)

\footnotetext{
${ }^{7}$ In health insurance, a copay (or copayment) is a set out-of-pocket amount paid by the insured for health care services. We use this terminology although our approach is not limited to health insurance. We assume, that the amounts paid in copays do not count towards meeting the deductible. In other words, the insurance indemnity is equal to the difference between the part of out-of-pocket costs over the copays and the deductible if this difference is positive.

${ }^{8}$ Using (4) then gives $w_{i j}^{00}-w_{i j}^{11}<2 L$.

${ }^{9}$ In such cases, $c_{i j}^{1}, c_{i j}^{2}$ and/or $D_{i j}$ are not uniquely defined as a function of $w_{i j}$. For instance, we have $T_{i j}^{10}=0, T_{i j}^{01}>0, T_{i j}^{11}>0$ when $w_{i j}^{00}-w_{i j}^{10}=L, w_{i j}^{00}-w_{i j}^{01}<L$ and $w_{i j}^{00}-w_{i j}^{11}<2 L$. Such an allocation may be sustained by $c_{i j}^{1}, c_{i j}^{2} \in[0, L]$ and $D_{i j} \geq 0$ such that $c_{i j}^{1}+D_{i j}>L, c_{i j}^{2}+D_{i j}<L$ and $c_{i j}^{1}+c_{i j}^{2}+D_{i j}>L$. This is possible under condition (4), by choosing $c_{i j}^{1}=w_{i j}^{01}-w_{i j}^{11}, c_{i j}^{2}+D_{i j}=w_{i j}^{00}-w_{i j}^{01}$ and $w_{i j}^{00}+w_{i j}^{11}-w_{i j}^{10}-w_{i j}^{01} \leq$ $D_{i j} \leq w_{i j}^{00}-w_{i j}^{01}$. In particular, $c_{i j}^{1}=w_{i j}^{01}-w_{i j}^{11}, c_{i j}^{2}=0, D_{i j}=w_{i j}^{00}-w_{i j}^{01}$ is a possible solution.
} 
may be sustained by an incentive scheme $T_{i j} \equiv\left(T_{i j}^{01}, T_{i j}^{10}, T_{i j}^{11}\right)$ such that

$$
T_{i j}^{x y}=\sup \left\{0, x\left(L-c_{i j}^{1}\right)+y\left(L-c_{i j}^{2}\right)-D_{i j}\right\},
$$

for $x, y \in\{0,1\}$.

Note that condition (4) may be rewritten as

$$
w_{i j}^{00}-w_{i j}^{11} \leq\left(w_{i j}^{00}-w_{i j}^{10}\right)+\left(w_{i j}^{00}-w_{i j}^{01}\right),
$$

which means that the loss $w_{i j}^{00}-w_{i j}^{11}$ retained by the household (i.e., not covered by insurance) in the case of two losses affecting simultaneously $r=1$ and $r=2$ is smaller than the sum of the retained losses $w_{i j}^{00}-w_{i j}^{10}$ and $w_{i j}^{00}-w_{i j}^{01}$ for the two events where a single loss affects either $r=1$ or $r=2$. In this sense, combining a deductible with copays provides a larger coverage for large risks than for small risks: the average indemnity per $\$$ loss is larger when losses affect both risk exposures simultaneously than when a single loss affects either $r=1$ or $r=2$ with equal probabilities. ${ }^{10}$

For illustrative purposes, Figures 1 and 2 show how changing the values of the deductible and of the copays allows the insurer to either cover more or less extensively the occurrence of two simultaneous losses or to prioritize a particular risk. Figure 1 illustrates the case where the coverage of large losses is reduced without affecting the coverage of small losses by increasing the deductible and decreasing the copays simultaneously. Figure 2 shows how the coverage of a single loss affecting risk $r=1$ can be reduced without changing the coverage of a single loss affecting $r=2$, and of a large loss affecting $r=1$ and $r=2$ simultaneously.

\section{Figure 1}

\section{Figure 2}

Consider now the case where the insurance scheme of type $i j$ households includes risk-specific copays $c_{i j}^{1}, c_{i j}^{2}$ and an out-of-pocket maximum $M_{i j}$ instead of an aggregate deductible. Let us focus on the case where $c_{i j}^{1}+c_{i j}^{2} \geq M_{i j}$

\footnotetext{
${ }^{10}$ In the case of two losses, the indemnity per dollar loss is $\left(w_{i j}^{00}-w_{i j}^{11}\right) / 2 L$. If there is one single loss affecting $r=1$ or $r=2$ with equal probabilities, the expected indemnity per dollar loss is $\left(w_{i j}^{00}-w_{i j}^{10}\right) / 2 L+\left(w_{i j}^{00}-w_{i j}^{01}\right) / 2 L$.
} 


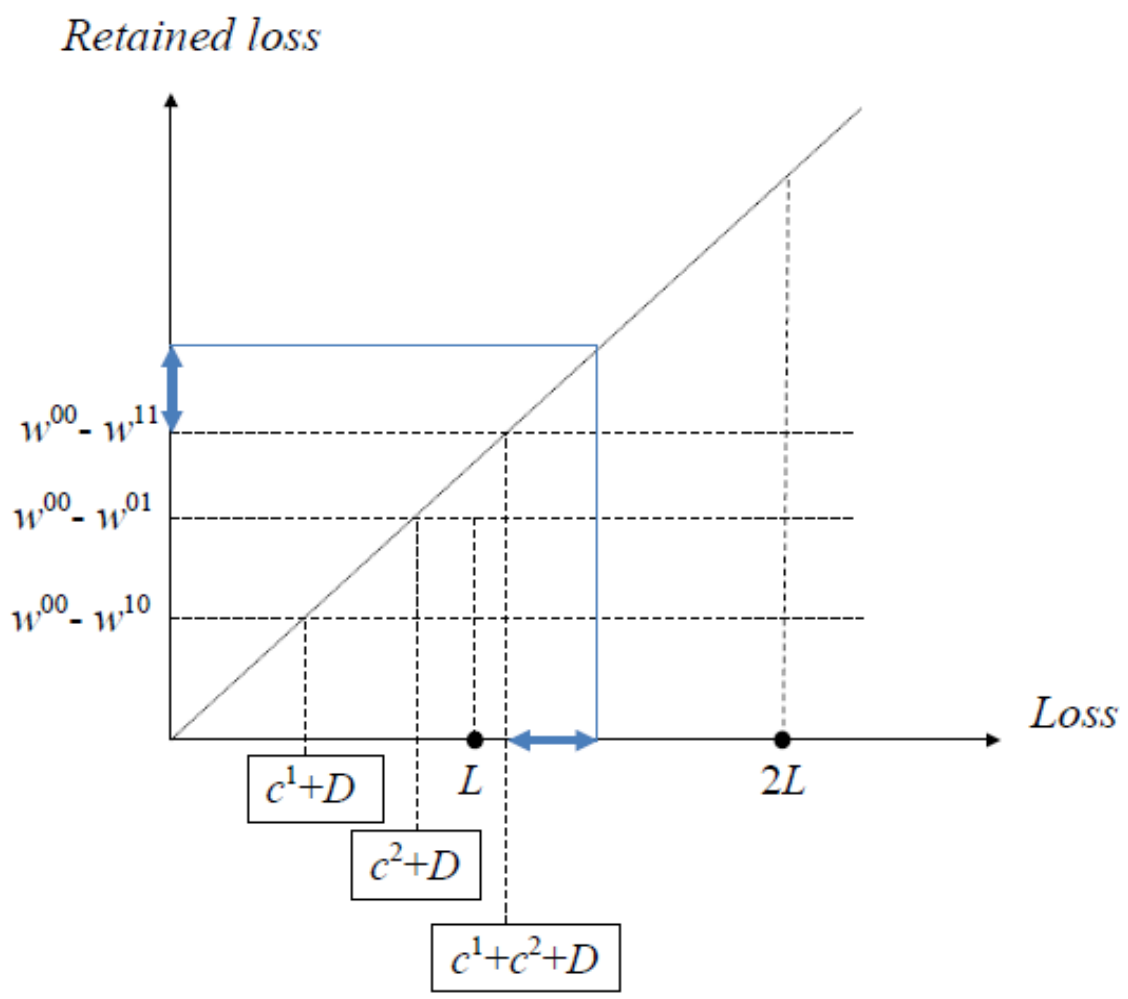

Figure 1: Increasing the deductible and decreasing the copays:

$$
\Delta D=-\Delta c^{1}=-\Delta c^{2}>0
$$




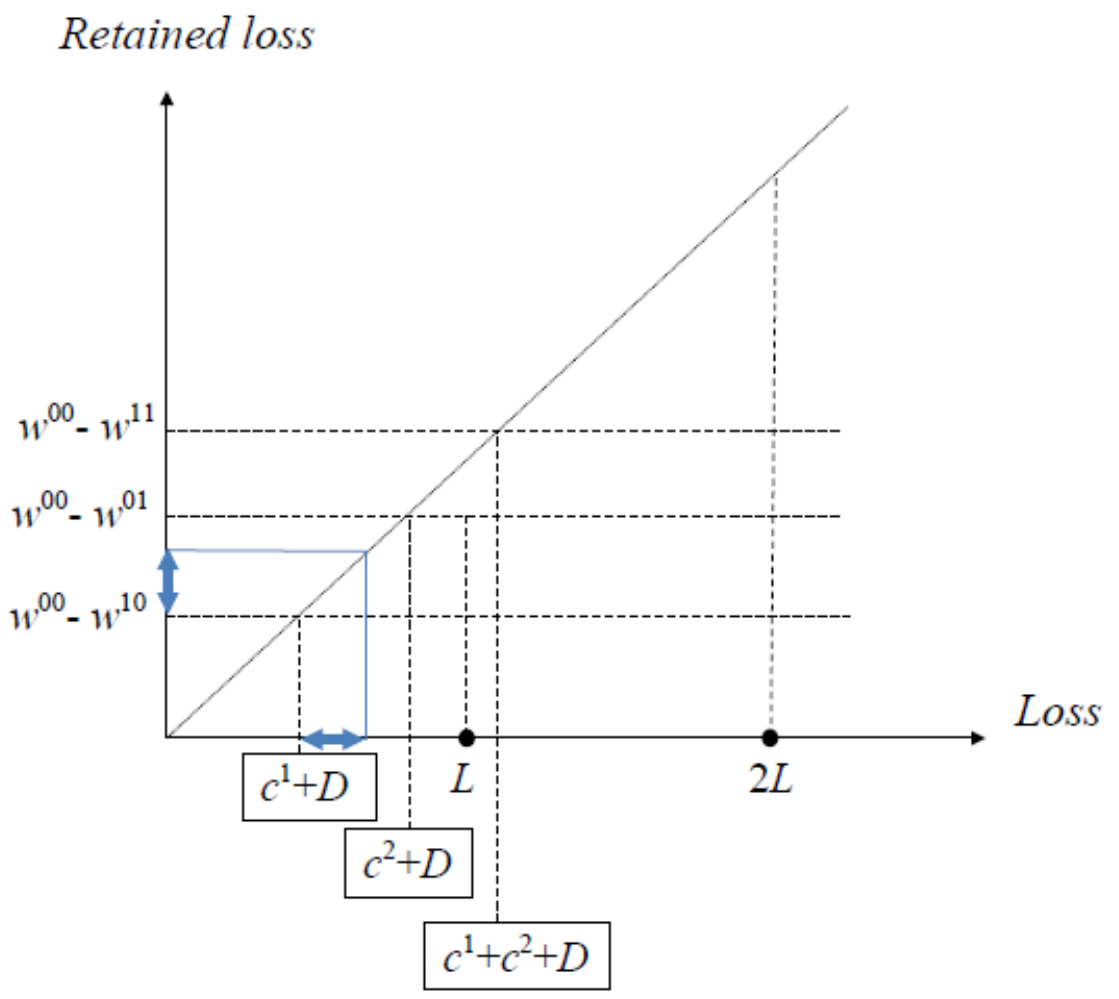

Figure 2: Increasing the deductible and decreasing the copay for $r=2$ :

$$
\Delta D=-\Delta c^{2}>0, \Delta c^{1}=0
$$


and $c_{i j}^{1}, c_{i j}^{1}<M_{i j}$, which means that the out-of-pocket maximum is reached in the case of two losses. In that case, we have

$$
\begin{aligned}
& T_{i j}^{10}=L-c_{i j}^{1}, \\
& T_{i j}^{01}=L-c_{i j}^{2}, \\
& T_{i j}^{11}=2 L-M_{i j},
\end{aligned}
$$

and (1) yields

$$
\begin{aligned}
& c_{i j}^{1}=w_{i j}^{00}-w_{i j}^{10}, \\
& c_{i j}^{2}=w_{i j}^{00}-w_{i j}^{01}, \\
& M_{i j}=w_{i j}^{00}-w_{i j}^{11} .
\end{aligned}
$$

Using $c_{i j}^{1}+c_{i j}^{2} \geq M_{i j}$ shows that $w_{i j}$ can be sustained by non-negative copays $c_{i j}^{1}, c_{i j}^{2}$ and an out-of-pocket maximum $M_{i j}$ if (4) holds, and thus, here also, we may conclude that mixing an out-of-pocket maximum with risk-specific copays allows the insurer to prioritize the coverage of large risks. Choosing $c_{i j}^{1}=L, c_{i j}^{2}=L$ and/or $M_{i j}=2 L$ leads to zero coverage when $T_{i j}^{01}, T_{i j}^{10}$ and/or $T_{i j}^{11}=0$, respectively. Overall, such an insurance scheme is defined by

$$
T_{i j}^{x y}=\sup \left\{x\left(L-c_{i j}^{1}\right)+y\left(L-c_{i j}^{2}\right), 2 L-M_{i j}\right\}
$$

for $x, y \in\{0,1\}$.

Finally, consider the case of risk-specific copays $c_{i j}^{1}$ and $c_{i j}^{2}$ combined with an upper limit on coverage $U L_{i j}$. The case where this upper limit is reached in the case of two losses corresponds to $L-c_{i j}^{1} \leq U L_{i j}, L-c_{i j}^{2} \leq U L_{i j}$ and $U L_{i j} \leq 2 L-c_{i j}^{1}-c_{i j}^{2}$. In that case, we have

$$
\begin{aligned}
& T_{i j}^{10}=L-c_{i j}^{1}, \\
& T_{i j}^{01}=L-c_{i j}^{2}, \\
& T_{i j}^{11}=U L_{i j},
\end{aligned}
$$

and (1) gives

$$
\begin{aligned}
& c_{i j}^{1}=w_{i j}^{00}-w_{i j}^{10}, \\
& c_{i j}^{2}=w_{i j}^{00}-w_{i j}^{01}, \\
& U L_{i j}=2 L+w_{i j}^{11}-w_{i j}^{00},
\end{aligned}
$$


with $U L_{i j} \leq 2 L-c_{i j}^{1}-c_{i j}^{2}$ if

$$
w_{i j}^{00}+w_{i j}^{11} \leq w_{i j}^{10}+w_{i j}^{01}
$$

which is the condition under which $w_{i j}$ can be sustained by non-negative copays $c_{i j}^{1}, c_{i j}^{2}$ and an upper limit on coverage $U L_{i j}$, with an interpretation which is symmetric to (4): mixing risk-specific copays with an upper limit on coverage is a way of prioritizing the coverage of losses that may affect one single risk exposure. Including the cases where no indemnity is paid yields

$$
T_{i j}^{x y}=\inf \left\{x\left(L-c_{i j}^{1}\right)+y\left(L-c_{i j}^{2}\right), U L_{i j}\right\}
$$

for $x, y \in\{0,1\}$.

\subsection{Incentive compatibility and feasibility}

The incentive compatibility of an allocation $\mathcal{A}=\left\{w_{h h}, w_{h \ell}, w_{\ell h}, w_{\ell \ell}\right\}$ requires that any type $i j$ household weakly prefers $w_{i j}$ to $w_{i^{\prime} j^{\prime}}$ if $i \neq i^{\prime}$ and $/$ or $j^{\prime} \neq j$, i.e.,

$$
U_{i j}\left(w_{i j}\right) \geq U_{i j}\left(w_{i^{\prime} j^{\prime}}\right),
$$

for all $i, j, i^{\prime}, j^{\prime} \in\{h, \ell\}$ such that $i \neq i^{\prime}$ and/or $j \neq j^{\prime}$.

Let $C_{i j}(w)$ be the expected wealth of a type $i j$ household, for statedependent wealth $w=\left(w^{00}, w^{01}, w^{10}, w^{11}\right)$, with

$$
\begin{aligned}
C_{h h}(w) \equiv & \left(1-p_{h}\right)^{2} w^{00}+p_{h}\left(1-p_{h}\right)\left(w^{01}+w^{10}\right)+p_{h}^{2} w^{11} \\
C_{h \ell}(w) \equiv & \left(1-p_{h}\right)\left(1-p_{\ell}\right) w^{00}+p_{\ell}\left(1-p_{h}\right) w^{01} \\
& +p_{h}\left(1-p_{\ell}\right) w^{10}+p_{h} p_{\ell} w^{11} \\
C_{\ell h}(w) \equiv & \left(1-p_{h}\right)\left(1-p_{\ell}\right) w^{00}+p_{h}\left(1-p_{\ell}\right) w^{01} \\
& +p_{\ell}\left(1-p_{h}\right) w^{10}+p_{h} p_{\ell} w^{11} \\
C_{\ell \ell}(w) \equiv & \left(1-p_{\ell}\right)^{2} w^{00}+p_{\ell}\left(1-p_{\ell}\right)\left(w^{01}+w^{10}\right)+p_{\ell}^{2} w^{11}
\end{aligned}
$$

By definition, the allocation $\mathcal{A}$ is budget-balanced if the average wealth in the population is lower or equal to the average wealth in the absence of any redistribution between households. This is written as

$$
\sum_{i, j \in\{h, \ell\}} \lambda_{i j} C_{i j}\left(w_{i j}\right) \leq \bar{w}
$$


where

$$
\bar{w}=w_{0}-L\left[2 \lambda_{h h} p_{h}+\left(\lambda_{h \ell}+\lambda_{\ell h}\right)\left(p_{h}+p_{\ell}\right)+2 \lambda_{\ell \ell} p_{\ell}\right] .
$$

Finally, we say that the allocation $\mathcal{A}$ is feasible if it is incentive compatible and budget-balanced.

Any feasible allocation can be induced by an insurance scheme where insurers offer menus of insurance schemes and make non-negative expected profit when households choose their best scheme. Indeed, assume that each type $i j$ household chooses a scheme $\left(T_{i j}, P_{i j}\right)$, among the offers available in the insurance market. In this case, the induced allocation $\mathcal{A}=\left\{w_{h h}, w_{h \ell}, w_{\ell h}, w_{\ell \ell}\right\}$ is defined by Equation (1) for all $i, j \in\{h, \ell\}$ and this allocation is incentive compatible. Conversely, any incentive compatible allocation $\mathcal{A}$ is induced by a menu of insurance contracts with coverage $T_{i} \equiv\left(T_{i j}^{01}, T_{i j}^{10}, T_{i j}^{11}\right)$ and premium $P_{i j}$ such that $P_{i j}=w_{i j}^{00}-w_{0}$ and $T_{i j}^{x y}=w_{i j}^{x y}-w_{i j}^{00}+(x+y) L$ if $(x, y) \neq(0,0)$.

Assume that insurers offer the menu of insurance schemes $\left\{\left(T_{i j}, P_{i j}\right), i, j=\right.$ $h, \ell\}$ and that $\left(T_{i j}, P_{i j}\right)$ is chosen by type $i j$ households in this menu. Assume moreover that policyholders are evenly spread among insurers. Then insurers make non-negative expected profit if

$$
\begin{aligned}
& \lambda_{h h}\left[P_{h h}-p_{h}\left(1-p_{h}\right)\left(T_{h h}^{01}+T_{h h}^{10}\right)-p_{h}^{2} T_{h h}^{11}\right] \\
& +\lambda_{h \ell}\left[P_{h \ell}-p_{\ell}\left(1-p_{h}\right) T_{h \ell}^{01}-p_{h}\left(1-p_{\ell}\right) T_{h \ell}^{10}-p_{h} p_{\ell} T_{h \ell}^{11}\right] \\
& +\lambda_{\ell h}\left[P_{\ell h}-p_{h}\left(1-p_{\ell}\right) T_{\ell h}^{01}-p_{\ell}\left(1-p_{h}\right) T_{\ell h}^{10}-p_{\ell} p_{h} T_{\ell h}^{11}\right] \\
& +\lambda_{\ell \ell}\left[P_{\ell \ell}-p_{\ell}\left(1-p_{\ell}\right)\left(T_{\ell \ell}^{01}+T_{\ell \ell}^{10}\right)-p_{\ell}^{2} T_{\ell \ell}^{11}\right] \\
& \geq 0
\end{aligned}
$$

$\mathcal{A}$ satisfies (7) if and only if (8) holds, which shows the correspondence between the insurers' break-even condition and the budget balance of allocations induced by insurance schemes. The results obtained so far are summarized in the following proposition.

Proposition 1 Conditions (6) and (7) define the set of feasible allocations that can be induced by insurance schemes offered by insurers that break even. Under condition (2) type ij households can be covered by risk-specific policies. Under condition (3) they can be covered by an umbrella policy. Under condition (4) - respect. condition (5) - they can be covered by an insurance policy that combines risk-specific copays with either a deductible or an out-of-pocket maximum - respect. an upper limit on indemnity -. 


\subsection{Second-best Pareto-optimality}

By definition, an allocation is second-best Pareto-optimal if it is feasible and if there does not exist another feasible allocation with expected utility at least as large for all types, and larger for at least one type. More explicitly, let us consider a feasible allocation $\mathcal{A}^{*}=\left\{w_{h h}^{*}, w_{h \ell}^{*}, w_{\ell h}^{*}, w_{\ell \ell}^{*}\right\}$, where $w_{i j}^{*}=$ $\left(w_{i j}^{00 *}, w_{i j}^{01 *}, w_{i j}^{10 *}, w_{i j}^{11 *}\right)$ for $i, j \in\{h, \ell\} . \mathcal{A}^{*}$ is second-best Pareto-optimal if, for all $i, j, U_{i j}\left(w_{i j}\right)$ reaches its maximum at $\mathcal{A}^{*}$ in the set of feasible allocations $\mathcal{A}=\left\{w_{h h}, w_{h \ell}, w_{\ell h}, w_{\ell \ell}\right\}$ such that $U_{i^{\prime} j^{\prime}}\left(w_{i^{\prime} j^{\prime}}\right) \geq U_{i^{\prime} j^{\prime}}\left(w_{i^{\prime} j^{\prime}}^{*}\right)$ for all $i^{\prime}, j^{\prime} \in\{h, \ell\}, i^{\prime} \neq i$ and/or $j^{\prime} \neq j$.

Investigating the competitive interactions that could lead to a particular second-best Pareto-optimal allocation is out of the scope of the present paper. In what follows, we will limit ourselves to analyzing the insurance contracts that sustain second-best Pareto-optimal allocations. Thus, we implicitly presume that, in the absence of trade restraint (such as barriers to entry or exogenous constraints on contracts), competitive forces should lead to a second-best Pareto optimal allocation, which is of course a debatable assumption. We may also have in mind a regulated insurance market where second-best Pareto-optimality results from public intervention, for instance through taxation of contracts.

\section{Risk splitting as an answer to adverse se- lection}

A second-best Pareto-optimal allocation $\mathcal{A}^{*}=\left\{w_{h h}^{*}, w_{h \ell}^{*}, w_{\ell h}^{*}, w_{\ell \ell}^{*}\right\}$ maximizes $U_{\ell \ell}\left(w_{\ell \ell}\right)$ in the set of feasible allocations $\mathcal{A}=\left\{w_{h h}, w_{h \ell}, w_{\ell h}, w_{\ell \ell}\right\}$ with expected utility equal to $u_{i j}^{*} \equiv U_{i j}\left(w_{i j}^{*}\right)$ for $i, j \in\{h, \ell\}, i \neq \ell$ and/or $j \neq \ell$. We also denote $u_{\ell \ell}^{*} \equiv U_{\ell \ell}\left(w_{\ell \ell}^{*}\right)$. Incentive compatibility implies that lower risk types reach weakly higher expected utility. ${ }^{11}$ We will assume

$$
u_{h h}^{*}<\min \left\{u_{h \ell}^{*}, u_{\ell h}^{*}\right\} \leq \max \left\{u_{h \ell}^{*}, u_{\ell h}^{*}\right\} \leq u_{\ell \ell}^{*},
$$

\footnotetext{
${ }^{11}$ This is true because overinsurance is excluded. In the Rothschild-Stiglitz (2016) setting, with only one risk exposure per type, second-best Pareto-optimal allocations exist where high-risk individuals are overcovered and reach a higher expected utility than lowrisk individuals, and such allocations could also exist in the present setting if overinsurance were allowed; See Crocker and Snow (1985).
} 
where the strict inequality $u_{h h}^{*}<\min \left\{u_{h \ell}^{*}, u_{\ell h}^{*}\right\}$ is made to exclude allocations where high-risk households are pooled with medium-risk households, i.e. where they have the same insurance coverage. ${ }^{12}$

Thus, $\mathcal{A}^{*}$ maximizes $U_{\ell \ell}\left(w_{\ell \ell}\right)$ with respect to $w_{h h}, w_{h \ell}, w_{\ell h}$ and $w_{\ell \ell}$, subject to

- Incentive compatibility constraints:

$$
U_{i j}\left(w_{i j}\right) \geq U_{i j}\left(w_{i^{\prime} j^{\prime}}\right)
$$

for all $i, j, i^{\prime}, j^{\prime} \in\{h, \ell\}$, with $i \neq i^{\prime}$ and/or $j \neq j^{\prime}$,

- Individual rationality constraints:

$$
U_{i j}\left(w_{i j}\right)=u_{i j}^{*}
$$

for all $i, j \in\{h, \ell\}$ with $i \neq \ell$ and/or $j \neq \ell$,

- Break-even constraint:

$$
\sum_{i, j \in\{h, \ell\}} \lambda_{i j} C_{i j}\left(w_{i j}\right) \leq \bar{w}
$$

and sign and no-overinsurance constraints.

This problem belongs to the class of multi-dimensional screening models surveyed by Armstrong and Rochet (1999) and Rochet and Stole (2003). Propositions 2 and 3 characterize its optimal solution, and the associated insurance schemes.

Proposition 2 Any second-best Pareto-optimal allocation is sustained by insurance schemes such that: (i) high-risk households are fully covered, (ii) medium-risk households are covered either by two risk-specific policies with full coverage for the large-risk exposure and partial coverage for the low-risk exposure, or only by a risk-specific policy with full coverage for the large-risk exposure, without coverage for the low-risk exposure, (iii) low-risk households are covered by a policy with partial coverage at the margin.

\footnotetext{
${ }^{12}$ Such pooling allocations may be second-best Pareto-optimal. For instance, in the standard Rothschild-Stiglitz model, the pooling allocation with full coverage at average actuarial price is a second-best Pareto-optimum. Our results can be extended to the case $u_{h h}^{*}=\min \left\{u_{h \ell}^{*}, u_{\ell h}^{*}\right\}$. Types $h h$ and $h \ell$ (respect. $h h$ and $\ell h$ ) would be pooled with the same coverage if $u_{h h}^{*}=u_{h \ell}^{*}$ (respect. if $u_{h h}^{*}=u_{\ell h}^{*}$ ). See the comments on Proposition 2.
} 


\section{Figure 3}

The proof of Proposition 2 is in two stages illustrated in Figure 3. At the first stage, we characterize the optimal solution of the relaxed problem where only the "weakly upward" incentive compatibility constraints $I C_{h h}^{h \ell}, I C_{h h}^{\ell h}, I C_{h h}^{\ell \ell}, I C_{h \ell}^{\ell h}, I C_{h \ell}^{\ell \ell}, I C_{\ell h}^{h \ell}$ and $I C_{\ell h}^{\ell \ell}$ are taken into account. At the second stage, we show that the optimal solution of the relaxed problem satisfies the incentive compatibility constraints that have been ignored at stage 1 and thus that it is the solution to the complete problem. ${ }^{13}$ This leads us to the following results:

$$
\begin{aligned}
& w_{h h}^{00}=w_{h h}^{10}=w_{h h}^{01}=w_{h h}^{11}, \\
& w_{h \ell}^{00}=w_{h \ell}^{10}>w_{h \ell}^{01}=w_{h \ell}^{11}, \\
& w_{\ell h}^{00}=w_{\ell h}^{01}>w_{\ell h}^{10}=w_{\ell h}^{11}, \\
& w_{\ell \ell}^{00}>\min \left\{w_{\ell \ell}^{01}, w_{\ell \ell}^{10}\right\} \\
& \operatorname{and} / \text { or } \\
& w_{\ell \ell}^{11}<\max \left\{w_{\ell \ell}^{01}, w_{\ell \ell}^{10}\right\} .
\end{aligned}
$$

Proposition 2 expresses these results in terms of insurance contracts. It states that households are covered by insurance contracts that depend on their risk type, at the same time regarding the size and contractual form of coverage. Equation (10) shows that, as in the Rothschild-Stiglitz (1976) model, high-risk households are fully covered, which means that $T_{h h}^{01}=T_{h h}^{10}=$ $L$ and $T_{h h}^{11}=2 L$. Indeed, the proof shows that the downward incentives constraints $I C_{h \ell}^{h h}, I C_{\ell h}^{h h}$ and $I C_{\ell \ell}^{h h}$ are not binding, and consequently there is no reason to distort the state-dependent wealth of high-risk households in order to prevent lower-risk households from choosing $w_{h h}$, instead of $w_{h \ell}, w_{\ell h}$ or $w_{\ell \ell}$. This is compatible either with an umbrella policy $\left(J_{h h}^{1}, J_{h h}^{2}, P_{h h}\right)$ such

\footnotetext{
${ }^{13}$ This approach is usual in adverse selection problems with the single-crossing condition and an arbitrary number of types. It is less obvious here, because there are four possible outcomes for each household, and not only two as in more standard models. See Armstrong and Rochet (1999) for an application of this approach to a principal-agent model with multi-dimensional information about the agent's type.
} 


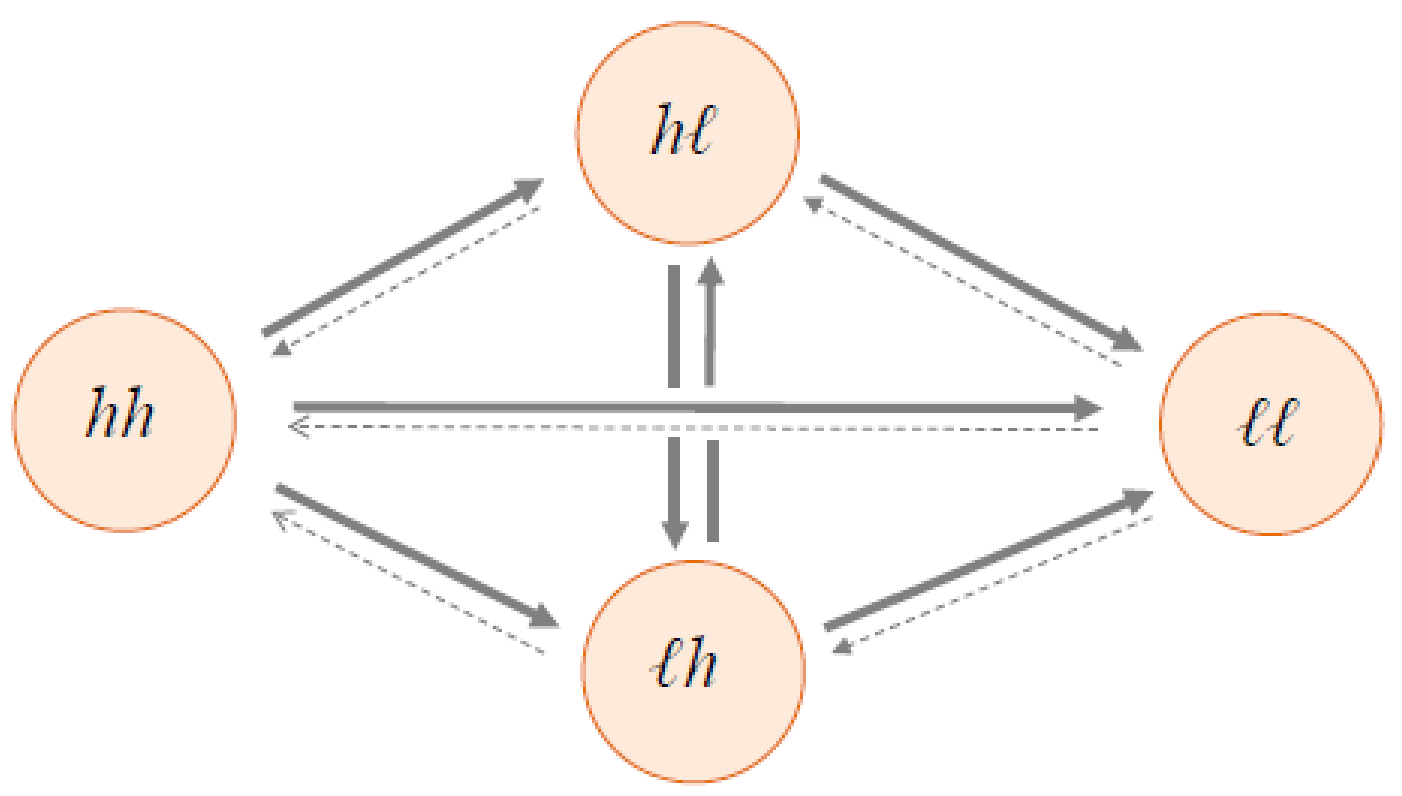

Figure 3

Incentive constraints that may be binding :

Non-binding incentive constraints: 
that $J_{h h}^{1}=L$ and $J_{h h}^{2}=2 L$, or with two risk-specific policies, $\left(I_{h h}^{1}, Q_{h h}^{1}\right)$ and $\left(I_{h h}^{2}, Q_{h h}^{2}\right)$, for risks $r=1$ and 2 , respectively, with $I_{h h}^{1}=I_{h h}^{2}=L$ and $Q_{h h}^{1}+Q_{h h}^{2}=P_{h h}$.

Equation (11) shows that medium-risk households are covered either by two risk-specific policies with full coverage for the high risk and partial coverage for the low risk, or by a single risk-specific policy with full coverage for the high risk, without coverage for the low risk. Hence, for type $h \ell$ households, we have $\left(I_{h \ell}^{1}, Q_{h \ell}^{1}\right)$ and $\left(I_{h \ell}^{2}, Q_{h \ell}^{2}\right)$ such that $I_{h \ell}^{1}=L$ and $0 \leq I_{h \ell}^{2}<L$, with $I_{h \ell}^{2}>0$ when $w_{h \ell}^{00}-w_{h \ell}^{01}<L$ and $I_{h \ell}^{2}=0$ (i.e. no coverage for the low risk) when $w_{h \ell}^{00}-w_{h \ell}^{01}=L$. The characterization is symmetric for type $\ell h$ households. This is not an astonishing conclusion if we have in mind the standard Rothschild-Stiglitz model, where the contract chosen by low-risk individuals provides partial coverage, which makes it unattractive to high-risk individuals. It is striking, and of course logical, that the same objective of reducing insurance compensation should lead insurers to offer risk-specific policies, where the low coverage (or even the absence of coverage) is for the low-risk exposure only. Reducing the compensation for the high-risk exposure would just be a waste of risk protection, without any effect on the separation of type $h \ell$ (or $\ell h$ ) from $h h$, since both are equally affected by this high risk. ${ }^{14,15}$

Finally, from equations (13) and (14), low-risk households are covered by an insurance scheme that provides partial coverage at the margin. More explicitly, we have partial coverage of a single loss affecting either $r=1$ or $r=2$ if $w_{\ell \ell}^{00}>\min \left\{w_{\ell \ell}^{01}, w_{\ell \ell}^{00}\right\}$. Likewise, we have partial coverage of a second loss when a first loss affects either $r=1$ or $r=2$, if $w_{\ell \ell}^{11}<\max \left\{w_{\ell \ell}^{01}, w_{\ell \ell}^{10}\right\}$. According to Proposition 3, low-risk households have partial coverage in at least one of these two cases.

The fact that low-risk households should be partially covered at the margin is not surprising, insofar as more risky households (i.e., types $h h, h \ell$ and eh) should be detered from choosing $w_{\ell \ell}$ and, as in the Rothschild-Stiglitz

\footnotetext{
${ }^{14}$ Providing partial coverage of the losses caused by risk exposure $r=2$ to type $h \ell$ individuals (i.e., choosing $w_{h \ell}^{01}<w_{h \ell}^{00}$ ) is a way to deter type $h h$ and $\ell h$ individuals from pretending that their type is $h \ell$. Maintaining full coverage for $r=1$ does not incentivize type $h h$ individuals to pretend that their type is $h \ell$, because $r=1$ is a high risk for $h h$ and $h \ell$. If $I C_{h h}^{h \ell}$ and $I C_{\ell h}^{h \ell}$ are both binding, then it would even be optimal to over-cover risk exposure $r=1$ of type $h \ell$ individuals (i.e. to choose $w_{h \ell}^{10}>w_{h \ell}^{00}$ ). Since over-coverage has been excluded, type $h \ell$ individual have full coverage for risk $r=1$.

${ }^{15}$ It can be checked that the strict inequalities in (11) and (12) become equalities if $u_{h \ell}^{*}=u_{h h}^{*}$ and $u_{h \ell}^{*}=u_{h h}^{*}$, respectively. In this case, medium risks are pooled with high risk policyholders and they have full coverage.
} 
model, this goes through partial coverage. Going beyond this conclusion requires additional assumptions, as shown in Proposition 3.

Proposition 3 At a second-best Pareto-optimal allocation, low-risk households are covered by an insurance scheme that combines risk-specific copays $c_{\ell \ell}^{1}, c_{\ell \ell}^{2}$ with a deductible $D_{\ell \ell}$, an out-of-pocket maximum $M_{\ell \ell}$ or an upper limit on coverage $U L_{\ell \ell}$. If households are prudent (i.e. $u^{\prime \prime \prime}>0$ ), then combining risk-specific copays with a deductible or with an out-of-pocket maximum is optimal when the fraction of high-risk households $\lambda_{h h}$ is small enough.

The low-risk state-dependent wealth $w_{\ell \ell}$ should reach a compromise between providing risk coverage to low-risk households and satisfying the incentive compatibility constraints of higher risk types, namely $I C_{h h}^{\ell \ell}, I C_{h \ell}^{\ell \ell}$ and $I C_{\ell h}^{\ell \ell}$. Intuitively, $I C_{h \ell}^{\ell \ell}$ will be all the easier, when the coverage of risk exposure $r=1$ is lower, i.e. when $c_{\ell \ell}^{1}$ is larger, because $r=1$ is a high risk for type $h \ell$ and a low risk for type $\ell \ell$. The conclusion is reversed for $I C_{\ell h}^{\ell \ell}$, with here a reason for increasing $c_{\ell \ell}^{2}$. Which copay should be the larger depends on the relative importance of types $h \ell$ and $\ell h$, including, among other things, their weights $\lambda_{h \ell}, \lambda_{\ell h}$ and expected utility levels $u_{h \ell}^{*}, u_{\ell h}^{*}$. The non-adjacent incentive compatibility constraint $I C_{h h}^{\ell \ell}$ does not justify treating risks $r=1$ and $r=2$ differently. Under the joint effect of adjacent and non-adjacent constraints, two cases are generically possible. If

$$
w_{\ell \ell}^{00}+w_{\ell \ell}^{11}>w_{\ell \ell}^{10}+w_{\ell \ell}^{01},
$$

then we know from condition (4) that risk-specific copays combined with either a deductible $D_{\ell \ell}$ or an out-of-pocket maximum $M_{\ell \ell}$ are optimal. On the contrary, if

$$
w_{\ell \ell}^{00}+w_{\ell \ell}^{11}<w_{\ell \ell}^{10}+w_{\ell \ell}^{01},
$$

then risk-specific copays combined with an upper limit on idemnity $U L_{\ell \ell}$ are optimal. Prudent policyholders are downside risk averse, and in that case, it seems intuitive that some priority should be given to the coverage of large losses. With the conclusions of section 2.2 in mind, we expect that (15) should hold, and thus that the optimal coverage of low-risk individuals should mix risk-specific copays with a deductible or an out-of-pocket maximum. However, this intuition is undermined when the $I C_{h h}^{\ell \ell}$ constraint is taken into account. Indeed, for a type $i j$ household, the probability $q_{i j}$ of having two 
losses, conditionally on the occurrence of at least one loss, is such that

$$
\begin{aligned}
q_{h h} & =\frac{p_{h}^{2}}{p_{h}\left(2-p_{h}\right)}, \\
q_{h \ell} & =q_{\ell h}=\frac{p_{h} p_{\ell}}{p_{h}+p_{\ell}-p_{h} p_{\ell}}, \\
q_{\ell \ell} & =\frac{p_{\ell}^{2}}{p_{\ell}\left(2-p_{\ell}\right)},
\end{aligned}
$$

with

$$
q_{\ell \ell}<q_{h \ell}=q_{\ell h}<q_{h h} .
$$

Thus, providing a generous coverage in the case of two losses is particularly attractive to high-risk households, and incentive constraint $I C_{h h}^{\ell \ell}$ justifies reducing the coverage of low-risk households in the case of two losses. In other words, for low-risk households, prudence favors the coverage of large losses, but deterring high-risk households from pretending that they are low risk goes in the opposite direction. ${ }^{16}$ Proposition 3 shows that the balance is tipped in favor of the coverage of large losses through a deductible or an outof-pocket maximum, mixed with risk-specific copays, when the proportion of high-risk households is small enough, so that they exert a relatively small effect on the optimal insurance coverage of low-risk households. ${ }^{17}$

\footnotetext{
${ }^{16}$ It may be objected that the same mechanism is also at work for medium-risk households, i.e. through constraints $I C_{h \ell}^{\ell \ell}$ and $I C_{\ell h}^{\ell \ell}$. This is true, but with a weaker intensity, so that this mechanism does not cancel the effect of downward risk aversion. See the proof of Proposition 3 for details.

${ }^{17}$ See the proof of Proposition 3 for a more explicit statement of the "for $\lambda_{h h}$ small enough" condition. Note that $u_{h h}^{*}, u_{h \ell}^{*}, u_{\ell h}^{*}, u_{\ell \ell}^{*}$ are considered as data, but in practice they result either from competitive market interactions and/or from public regulation. Whatever the mechanism we may have in mind, it will not determine $u_{h h}^{*}, u_{h \ell}^{*}, u_{\ell h}^{*}, u_{\ell \ell}^{*}$ independently from $\lambda_{h h}, \lambda_{h \ell}, \lambda_{\ell h}, \lambda_{\ell \ell}$. Hence, the second part of Proposition 3 should not be overinterpreted: it allows us to understand the mechanism that leads to a specific form of coverage, rather than explaining what is expected to occur in markets where the fraction of small-risk households is small.
} 


\section{On the role of transaction costs}

Let us now consider how transaction costs affect our conclusions. We assume that insurance premiums should also cover such transaction costs, under the form of proportional loading at rate $\sigma$. Hence, insurers make non-negative expected profit if

$$
\begin{aligned}
& \lambda_{h h}\left\{P_{h h}-(1+\sigma)\left[p_{h}\left(1-p_{h}\right)\left(T_{h h}^{01}+T_{h h}^{10}\right)+p_{h}^{2} T_{h h}^{11}\right]\right\} \\
& +\lambda_{h \ell}\left\{P_{h \ell}-(1+\sigma)\left[p_{\ell}\left(1-p_{h}\right) T_{h \ell}^{01}-p_{h}\left(1-p_{\ell}\right) T_{h \ell}^{10}+p_{h} p_{\ell} T_{h \ell}^{11}\right]\right\} \\
& +\lambda_{\ell h}\left\{P_{\ell h}-(1+\sigma)\left[p_{h}\left(1-p_{\ell}\right) T_{\ell h}^{01}-p_{\ell}\left(1-p_{h}\right) T_{\ell h}^{10}+p_{\ell} p_{h} T_{\ell h}^{11}\right]\right\} \\
& +\lambda_{\ell \ell}\left\{P_{\ell \ell}-(1+\sigma)\left[p_{\ell}\left(1-p_{\ell}\right)\left(T_{\ell \ell}^{01}+T_{\ell \ell}^{10}\right)+p_{\ell}^{2} T_{\ell \ell}^{11}\right]\right\} \\
& \geq 0,
\end{aligned}
$$

instead of (8). Using (1) shows that the budget balance condition may still be written as equation (7), after rewriting the households' expected wealth as

$$
\begin{aligned}
C_{h h}(w) \equiv & {\left[1-(1+\sigma) p_{h}\left(2-p_{h}\right)\right] w^{00}+(1+\sigma) p_{h}\left(1-p_{h}\right)\left(w^{01}+w^{10}\right) } \\
& +(1+\sigma) p_{h}^{2} w^{11} \\
C_{h \ell}(w) \equiv & {\left[1-(1+\sigma)\left(p_{h}+p_{\ell}-p_{h} p_{\ell}\right)\right] w^{00}+(1+\sigma)\left[p_{\ell}\left(1-p_{h}\right) w^{01}\right.} \\
& \left.+p_{h}\left(1-p_{\ell}\right) w^{10}\right]+(1+\sigma) p_{h} p_{\ell} w^{11}, \\
C_{\ell h}(w) \equiv & {\left[1-(1+\sigma)\left(p_{h}+p_{\ell}-p_{h} p_{\ell}\right)\right] w^{00}+(1+\sigma)\left[p_{h}\left(1-p_{\ell}\right) w^{01}\right.} \\
& \left.+p_{\ell}\left(1-p_{h}\right) w^{10}\right]+(1+\sigma) p_{\ell} p_{h} w^{11} \\
C_{\ell \ell}(w) \equiv & {\left[1-(1+\sigma) p_{\ell}\left(2-p_{\ell}\right)\right] w^{00}+(1+\sigma) p_{\ell}\left(1-p_{\ell}\right)\left(w^{01}+w^{10}\right) } \\
& +(1+\sigma) p_{\ell}^{2} w^{11}
\end{aligned}
$$

and with

$$
\bar{w}=w_{0}-L(1+\sigma)\left[2 \lambda_{h h} p_{h}+\left(\lambda_{h \ell}+\lambda_{\ell h}\right)\left(p_{h}+p_{\ell}\right)+2 \lambda_{\ell \ell} p_{\ell}\right] .
$$

Obviously a large value of $\sigma$ may make insurance unattractive. In order to focus attention on cases where there is still some scope for insurance, we assume that $\sigma$ is not overly large, so that any risk that would be fully covered if $\sigma=0$ is still at least partially covered. ${ }^{18}$ Proposition 4 characterizes optimal

\footnotetext{
${ }^{18}$ Mor explicitly, for all $i, j \in\{h, \ell\}$ and all $x, y \in\{0,1\}, \sigma$ is such that $T_{i j}^{x 1}>T_{i j}^{x 0}$ and $T_{i j}^{1 y}>T_{i j}^{0 y}$ if $T_{i j}^{x 1}=T_{i j}^{x 0}+L$ and $T_{i j}^{1 y}=T_{i j}^{0 y}+L$, respectively, when $\sigma=0$
} 
insurance schemes in this setting, by focusing attention on the optimality of deductibles combined with copays. ${ }^{19}$

Proposition 4 If there are transaction costs under the form of proportional loading, then any second-best Pareto-optimal allocation is sustained by insurance schemes such that: ( $i$ ) high-risk households are covered by a straight deductible policy (i.e. a policy with a deductible and no copay). (ii) medium risk households are covered either by a single policy with a deductible and a copay for the low risk, or by two risk-specific policies with full coverage for the high risk and partial coverage for the low risk, or partial coverage for the high risk and no coverage for the low risk. (iii) low-risk households are covered by a single policy with a deductible and risk-specific copays if households are prudent and the fraction of high-risk households is small enough.

Proposition 4 starts with the optimality of straight deductible contracts for high risk households. This is written as

$$
T_{h h}^{x y}=\sup \left\{0,(x+y) L-D_{h h}\right\},
$$

where $D_{h h}$ is the deductible of type $h h$ households. In other words, comparing Proposition 4 with Proposition 2 shows that full coverage is just replaced by a straight deductible as in usual insurance demand models with proportional loading. There is nothing astonishing here, since incentive compatibility constraints do not justify introducing distortions between risk exposures 1 and 2 in the high-risk state-dependent wealth.

According to Proposition 4, medium-risk households should be covered either by an insurance policy that bundles the two risk exposures with a deductible and a copay for the low risk, or by risk specific policies with full coverage for the high risk and partial coverage for the low risk, or with partial coverage for the high risk and zero coverage for the low risk. In the case of type $h \ell$ households, these indemnity schedules are written as

$$
T_{h \ell}^{x y}=\sup \left\{0, x L+y\left(L-c_{h \ell}^{2}\right)-D_{h \ell}\right\},
$$

\footnotetext{
${ }^{19}$ The optimality of straight deductible contracts when there is loading and no asymmetric information justifies the special attention put on deductibles. However, in our setting, out-of-pocket maxima and deductibles, combined with copays, may be used to sustain the same allocations.
} 
in the case of a deductible $D_{h \ell}$ and copay $c_{h \ell}^{2}$ for $r=2$, and

$$
T_{h \ell}^{10}=I_{h \ell}^{1}, T_{h \ell}^{01}=I_{h \ell}^{2}, T_{h \ell}^{11}=I_{h \ell}^{1}+I_{h \ell}^{2},
$$

with either $I_{h \ell}^{1}=L, I_{h \ell}^{2}<L$ or $I_{h \ell}^{1}<L, I_{h \ell}^{2}=0$, in the case of risk-specific policies. A symmetric characterization is obtained for type $\ell h$ households.

The intuition for this characterization is as follows. Note firstly that, as in Proposition 2, separating type $h \ell$ from types $h h$ and $\ell h$ requires risk exposure $r=1$ to be more extensively covered than risk exposure $r=2$. However, insurance loading is a reason for decreasing the coverage, whether caused by risk exposure $r=1$ or 2 . We know from Proposition 2 that, when there is no loading, risk-specific policies are optimal with full coverage for the high risk and partial or even zero coverage for the low risk. As illustrated in formula (19), substracting the deductible $D_{h \ell}$ from $x L+y\left(L-c_{h \ell}^{2}\right)$ is a way of reducing the indemnity payment uniformly, as long as it is positive, whatever the origin of the loss, while maintaining a larger coverage for $r=1$ than for $r=2$. Hence, combining a deductible with a copay for the low risk is a natural way to preserve incentives, while spreading the weight of transaction costs over the two sources of risk.

If $I C_{h h}^{h \ell}$ is the only incentive compatibility constraint that affects the state contingent allocation $w_{h \ell}$, then there is no reason to distort the coverage of risk exposure $r=1$, because it is a high risk for both type $h h$ and $h \ell$ households: in this case, substracting the deductible $D_{h \ell}$ from the aggregate coverage $x L+y\left(L-c_{h \ell}^{2}\right)$ is optimal. However, if $I C_{\ell h}^{h \ell}$ is also binding, then it may be optimal to maintain a full coverage for $r=1$ and to reduce the coverage for $r=2$ when $\sigma>0 .^{20}$ In other words, under the simultaneous effects of the two incentive compatibility constraints $I C_{h h}^{h \ell}$ and $I C_{\ell h}^{h \ell}$, it may be optimal to concentrate the decrease in coverage due to transaction costs on the low-risk exposure. This may even lead to fully cancelling the coverage of the low-risk exposure and, in that case, the incentives are reinforced by providing partial coverage for the high-risk exposure. In these cases, riskspecific policies are still optimal for medium-risk households, as in the no transaction cost case.

\footnotetext{
${ }^{20}$ Intuitively, if $I C_{\ell h}^{h \ell}$ is binding, then providing overinsurance for $r=1$ to type $h \ell$ households would relax the weight of this constraint, with a zero first-order effect in the neighbourhood of full insurance. We have excluded overinsurance, but constraint $I C_{\ell h}^{h \ell}$ may justify keeping $r=1$ fully covered for type $h \ell$ households when $\sigma$ is positive and not too large.
} 
Finally, there is no change for type $\ell \ell$ households: if households are prudent and high-risk households correspond to a small proportion of the population, then low-risk households are covered by an insurance policy that bundles the two risk exposures, and includes a deductible $D_{\ell \ell}$ and risk-specific copays $c_{\ell \ell}^{1}, c_{\ell \ell}^{2}$. This is written as

$$
T_{\ell \ell}^{x y}=\sup \left\{0, x\left(L-c_{\ell \ell}^{1}\right)+y\left(L-c_{\ell \ell}^{2}\right)-D_{\ell \ell}\right\},
$$

where the relative size of the copays reflect the intensity of the incentive pressures of the two types of medium-risk households. Hence, for low-risk households, loading affects the size of the deductible, but not the structure of the indemnity schedule, with specific indemnity rules for each risk exposure.

\section{Conclusion}

Although risk splitting is ubiquitous in insurance markets, economic theory lacks arguments to explain why, more often than not, policyholders are not covered by umbrella policies. In this paper, we have pursued the idea that, under adverse selection, risk splitting facilitates the separation of risk types, and thus that risk-specific indemnity rules should be part and parcel of insurance policies, except for high-risk households. By doing so, we have established that either risk-specific insurance policies or the combination of risk-specific copays with a deductible, an out-of-pocket maximum, or an upper limit on coverage are in fact optimal answers to the adverse selection challenge.

Much work remains to be done to reach a fully satisfactory understanding of the structure of insurance contracts when policyholders face multiple risks. Future research in this field could consider a setting that would be less restrictive than the one contemplated in this paper, with more than two risk exposures, one level of loss per risk and two risk types. Moreover, it would be worth exploring alternative explanations of risk splitting, whether they are associated with other forms of asymmetry of information between insurer and insured or with managerial constraints. Finally, and most importantly, we have only explained why risk splitting is inherently linked to second-best efficiency, but we have not analyzed how market strategies may actually lead to the implemention of such insurance contracts. Understanding how competition forces interact under adverse selection when policyholders face multiple risks is of prime importance. It has to be noted that the analysis of insurance 
markets under adverse selection has not reached a consensus in the case of one single risk per policyholder ${ }^{21}$ and undoubtedly, considering multiple risk exposures is an additional challenge for this line of research.

${ }^{21}$ See the survey of Mimra and Wambach (2014). 


\section{Appendix 1: Proofs}

\section{Proof of Proposition 2}

Consider a second-best Pareto optimal allocation with expected utility $u_{i j}^{*}$ for type $i j$ households, with $i, j \in\{h, \ell\}$. This allocation maximizes

$$
U_{\ell \ell}\left(w_{\ell \ell}\right) \equiv\left(1-p_{\ell}\right)^{2} u\left(w_{\ell \ell}^{00}\right)+\left(1-p_{\ell}\right) p_{\ell}\left[u\left(w_{\ell \ell}^{01}\right)+u\left(w_{\ell \ell}^{10}\right)\right]+p_{\ell}^{2} u\left(w_{\ell \ell}^{11}\right)
$$

with respect to $w_{h h}, w_{h \ell}, w_{\ell h}, w_{\ell \ell}$, subject to:

- Incentive compatibility constraints:

$$
\begin{aligned}
& \left(1-p_{h}\right)^{2} u\left(w_{h h}^{00}\right)+p_{h}\left(1-p_{h}\right)\left[u\left(w_{h h}^{01}\right)+u\left(w_{h h}^{10}\right)\right]+p_{h}^{2} u\left(w_{h h}^{11}\right) \\
& \geq \\
& \left(1-p_{h}\right)^{2} u\left(w_{h \ell}^{00}\right)+p_{h}\left(1-p_{h}\right)\left[u\left(w_{h \ell}^{01}\right)+u\left(w_{h \ell}^{10}\right)\right]+p_{h}^{2} u\left(w_{h \ell}^{11}\right),
\end{aligned}
$$

with a similar inequation for $\left(I C_{h h}^{\ell h}\right)$,

$$
\begin{gathered}
\left(1-p_{h}\right)^{2} u\left(w_{h h}^{00}\right)+p_{h}\left(1-p_{h}\right)\left[u\left(w_{h h}^{01}\right)+u\left(w_{h h}^{10}\right)\right]+p_{h}^{2} u\left(w_{h h}^{11}\right) \\
\geq \\
\left(1-p_{h}\right)^{2} u\left(w_{\ell \ell}^{00}\right)+p_{h}\left(1-p_{h}\right)\left[u\left(w_{\ell \ell}^{01}\right)+u\left(w_{\ell \ell}^{10}\right)\right]+p_{h}^{2} u\left(w_{\ell \ell}^{11}\right), \quad\left(I C_{h h}^{\ell \ell}\right) \\
\left(1-p_{h}\right)\left(1-p_{\ell}\right) u\left(w_{h \ell}^{00}\right)+p_{\ell}\left(1-p_{h}\right) u\left(w_{h \ell}^{01}\right)+p_{h}\left(1-p_{\ell}\right) u\left(w_{h \ell}^{10}\right)+p_{h} p_{\ell} u\left(w_{h \ell}^{11}\right) \\
\geq \\
\left.\left(1-p_{h}\right)\left(1-p_{\ell}\right) u\left(w_{h h}^{00}\right)+p_{\ell}\left(1-p_{h}\right) u\left(w_{h h}^{01}\right)+p_{h}\left(1-p_{\ell}\right) u\left(w_{h h}^{10}\right)\right]+p_{h} p_{\ell} u\left(w_{h h}^{11}\right), \\
\left(I C_{h \ell}^{h h}\right)
\end{gathered}
$$

with a similar inequation for $\left(I C_{\ell h}^{h h}\right)$,

$$
\begin{aligned}
& \left(1-p_{h}\right)\left(1-p_{\ell}\right) u\left(w_{h \ell}^{00}\right)+p_{\ell}\left(1-p_{h}\right) u\left(w_{h \ell}^{01}\right)+p_{h}\left(1-p_{\ell}\right) u\left(w_{h \ell}^{10}\right)+p_{h} p_{\ell} u\left(w_{h \ell}^{11}\right) \\
& \geq \\
& \left.\left(1-p_{h}\right)\left(1-p_{\ell}\right) u\left(w_{\ell h}^{00}\right)+p_{\ell}\left(1-p_{h}\right) u\left(w_{\ell h}^{01}\right)+p_{h}\left(1-p_{\ell}\right) u\left(w_{\ell h}^{10}\right)\right]+p_{h} p_{\ell} u\left(w_{\ell h}^{11}\right), \\
& \left(I C_{h \ell}^{\ell h}\right)
\end{aligned}
$$


with a similar inequation for $\left(I C_{\ell h}^{h \ell}\right)$,

$$
\begin{aligned}
& \left(1-p_{h}\right)\left(1-p_{\ell}\right) u\left(w_{h \ell}^{00}\right)+p_{\ell}\left(1-p_{h}\right) u\left(w_{h \ell}^{01}\right)+p_{h}\left(1-p_{\ell}\right) u\left(w_{h \ell}^{10}\right)+p_{h} p_{\ell} u\left(w_{h \ell}^{11}\right) \\
& \geq \\
& \left.\left(1-p_{h}\right)\left(1-p_{\ell}\right) u\left(w_{\ell \ell}^{00}\right)+p_{\ell}\left(1-p_{h}\right) u\left(w_{\ell \ell}^{01}\right)+p_{h}\left(1-p_{\ell}\right) u\left(w_{\ell \ell}^{10}\right)\right]+p_{h} p_{\ell} u\left(w_{\ell \ell}^{11}\right), \\
& \left(I C_{h \ell}^{\ell \ell}\right)
\end{aligned}
$$

with a similar inequation for $\left(I C_{\ell h}^{\ell \ell}\right)$,

$$
\begin{aligned}
& \left(1-p_{\ell}\right)^{2} u\left(w_{\ell \ell}^{00}\right)+\left(1-p_{\ell}\right) p_{\ell}\left[u\left(w_{\ell \ell}^{01}\right)+u\left(w_{\ell \ell}^{10}\right)\right]+p_{\ell}^{2} u\left(w_{\ell \ell}^{11}\right) \\
& \geq \\
& \left(1-p_{\ell}\right)^{2} u\left(w_{h h}^{00}\right)+\left(1-p_{\ell}\right) p_{\ell}\left[u\left(w_{h h}^{01}\right)+u\left(w_{h h}^{10}\right)\right]+p_{\ell}^{2} u\left(w_{h h}^{11}\right), \quad\left(I C_{\ell \ell}^{h h}\right) \\
& \left(1-p_{\ell}\right)^{2} u\left(w_{\ell \ell}^{00}\right)+\left(1-p_{\ell}\right) p_{\ell}\left[u\left(w_{\ell \ell}^{01}\right)+u\left(w_{\ell \ell}^{10}\right)\right]+p_{\ell}^{2} u\left(w_{\ell \ell}^{11}\right) \\
& \geq \\
& \left(1-p_{\ell}\right)^{2} u\left(w_{h \ell}^{00}\right)+\left(1-p_{\ell}\right) p_{\ell}\left[u\left(w_{h \ell}^{01}\right)+u\left(w_{h \ell}^{10}\right)\right]+p_{\ell}^{2} u\left(w_{h \ell}^{11}\right),
\end{aligned}
$$

with a similar inequation for $\left(I C_{\ell \ell}^{\ell h}\right)$,

\section{- Individual rationality constraints:}

$$
\begin{aligned}
U_{h h}\left(w_{h h}\right) \equiv & \left(1-p_{h}\right)^{2} u\left(w_{h h}^{00}\right)+\left(1-p_{h}\right) p_{h}\left[u\left(w_{h h}^{01}\right)+u\left(w_{h h}^{10}\right)\right] \\
& +p_{h}^{2} u\left(w_{h h}^{11}\right)=u_{h h}^{*}, \\
U_{h \ell}\left(w_{h \ell}\right) \equiv & \left(1-p_{h}\right)\left(1-p_{\ell}\right) u\left(w_{h \ell}^{00}\right)+p_{\ell}\left(1-p_{h}\right) u\left(w_{h \ell}^{01}\right) \\
& +p_{h}\left(1-p_{\ell}\right) u\left(w_{h \ell}^{10}\right)+p_{h} p_{\ell} u\left(w_{h \ell}^{11}\right)=u_{h \ell}^{*},
\end{aligned}
$$

with a similar equation for $\left(I R_{\ell h}\right)$, and subject to the break-even constraint $(B C)$, to non-over insurance constraints:

$$
\begin{array}{lll}
w_{i j}^{01} \leq w_{i j}^{00}, & & \left(N O I_{i j}^{00 / 01}\right) \\
w_{i j}^{10} \leq w_{i j}^{00}, & & \left(N O I_{i j}^{00 / 10}\right) \\
w_{i j}^{11} \leq w_{i j}^{01}, & & \left(N O I_{i j}^{01 / 11}\right) \\
w_{i j}^{11} \leq w_{i j}^{10}, & & \left(N O I_{i j}^{10 / 11}\right)
\end{array}
$$


for $i, j \in\{h, \ell\}$, and to non-negative indemnity constraints:

$$
\begin{aligned}
w_{i j}^{00}-w_{i j}^{01} \leq L, & & \left(N N I_{i j}^{00 / 01}\right) \\
w_{i j}^{10}-w_{i j}^{11} \leq L, & & \left(N N I_{i j}^{10 / 11}\right) \\
w_{i j}^{00}-w_{i j}^{10} \leq L, & & \left(N N I_{i j}^{00 / 10}\right) \\
w_{i j}^{01}-w_{i j}^{11} \leq L, & & \left(N N I_{i j}^{01 / 11}\right)
\end{aligned}
$$

for $i, j \in\{h, \ell\}$. The rest of the proof is in two stages.

\section{Stage 1}

Let us characterize the optimal solution to the relaxed problem, in which we delete the downward incentive compatibility constraints $I C_{\ell \ell}^{h \ell}, I C_{\ell \ell}^{\ell h}, I C_{h \ell}^{h h}$, $I C_{\ell h}^{h h}$ and $I C_{\ell \ell}^{h h}$, the no-overinsurance constraints except $N O I_{h \ell}^{00 / 10}, N O I_{h \ell}^{01 / 11}$, $N O I_{\ell h}^{00 / 01}, N O I_{\ell h}^{10 / 11}, N O I_{\ell \ell}^{00 / 01}, N O I_{\ell \ell}^{00 / 10}, N O I_{\ell \ell}^{01 / 11}, N O I_{\ell \ell}^{10 / 11}$, and the nonnegative indemnity constraints except $N N I_{h \ell}^{00 / 01}, N N I_{h \ell}^{10 / 11}, N N I_{\ell h}^{00 / 10}, N N I_{\ell h}^{01 / 11}$, $N N I_{\ell \ell}^{00 / 01}, N N I_{\ell \ell}^{00 / 10}, N N I_{\ell \ell}^{01 / 11}, N N I_{\ell \ell}^{10 / 11}$.

Let

$$
\begin{aligned}
& \alpha_{h h}^{h \ell}, \alpha_{h h}^{\ell h}, \alpha_{h h}^{\ell \ell}, \alpha_{h \ell}^{\ell h}, \alpha_{h \ell}^{\ell \ell}, \alpha_{\ell h}^{h \ell}, \alpha_{\ell h}^{\ell \ell} \geq 0 \\
& \delta_{h \ell}^{00 / 10}, \delta_{h \ell}^{01 / 11}, \delta_{\ell h}^{00 / 01}, \delta_{\ell h}^{10 / 11}, \delta_{\ell \ell}^{00 / 01} \geq 0, \\
& \delta_{\ell \ell}^{00 / 10}, \delta_{\ell \ell}^{01 / 11}, \delta_{\ell \ell}^{10 / 11}, \gamma \geq 0, \\
& \eta_{h \ell}^{00 / 01}, \eta_{h \ell}^{10 / 11}, \eta_{\ell h}^{00 / 10}, \eta_{\ell h}^{01 / 11}, \geq 0, \\
& \eta_{\ell \ell}^{00 / 01}, \eta_{\ell \ell}^{00 / 10}, \eta_{\ell \ell}^{01 / 11}, \eta_{\ell \ell}^{10 / 11} \geq 0, \\
& \beta_{h h}, \beta_{h \ell}, \beta_{\ell h},
\end{aligned}
$$

be Lagrange multipliers associated with

$$
\begin{aligned}
& I C_{h h}^{h \ell}, I C_{h h}^{\ell h}, I C_{h h}^{\ell \ell}, I C_{h \ell}^{\ell h}, I C_{h \ell}^{\ell \ell}, I C_{\ell h}^{h \ell}, I C_{\ell h}^{\ell \ell}, \\
& N O I_{h \ell}^{00 / 10}, N O I_{h \ell}^{01 / 11}, N O I_{\ell h}^{00 / 01}, N O I_{\ell h}^{10 / 11}, N O I_{\ell \ell}^{00 / 01}, \\
& N O I_{\ell \ell}^{00 / 10}, N O I_{\ell \ell}^{01 / 11}, N O I_{\ell \ell}^{10 / 11}, B C, \\
& N N I_{h \ell}^{00 / 01}, N N I_{h \ell}^{10 / 11}, N N I_{\ell h}^{00 / 10}, N N I_{\ell h}^{01 / 11}, \\
& N N I_{\ell \ell}^{00 / 01}, N N I_{\ell \ell}^{00 / 10}, N N I_{h \ell}^{01 / 11}, N N I_{h \ell}^{10 / 11} \\
& I R_{h h}, I R_{h \ell}, I R_{\ell h},
\end{aligned}
$$


respectively.

A preliminary step of the proof consists in showing that $\gamma>0 .^{22}$ This is done in Lemma 1, put at the end of this proof. Let $\mathcal{L}$ be the corresponding Lagrangian. The first-order optimality conditions are written as:

$$
\begin{gathered}
\frac{\partial \mathcal{L}}{\partial w_{h h}^{00}}=\left(1-p_{h}\right)^{2}\left[\left(\alpha_{h h}^{h \ell}+\alpha_{h h}^{\ell h}+\alpha_{h h}^{\ell \ell}+\beta_{h h}\right) u^{\prime}\left(w_{h h}^{00}\right)-\gamma \lambda_{h h}\right]=0 \\
\frac{\partial \mathcal{L}}{\partial w_{h h}^{01}}=p_{h}\left(1-p_{h}\right)\left[\left(\alpha_{h h}^{h \ell}+\alpha_{h h}^{\ell h}+\alpha_{h h}^{\ell \ell}+\beta_{h h}\right) u^{\prime}\left(w_{h h}^{01}\right)-\gamma \lambda_{h h}\right]=0 \\
\frac{\partial \mathcal{L}}{\partial w_{h h}^{10}}=p_{h}\left(1-p_{h}\right)\left[\left(\alpha_{h h}^{h \ell}+\alpha_{h h}^{\ell h}+\alpha_{h h}^{\ell \ell}+\beta_{h h}\right) u^{\prime}\left(w_{h h}^{10}\right)-\gamma \lambda_{h h}\right]=0 \\
\frac{\partial \mathcal{L}}{\partial w_{h h}^{11}}=p_{h}^{2}\left[\left(\alpha_{h h}^{h \ell}+\alpha_{h h}^{\ell h}+\alpha_{h h}^{\ell \ell}+\beta_{h h}\right) u^{\prime}\left(w_{h h}^{11}\right)-\gamma \lambda_{h h}\right]=0 \\
\frac{\partial \mathcal{L}}{\partial w_{h \ell}^{00}}=\left(1-p_{h}\right)\left(1-p_{\ell}\right)\left[\left(\alpha_{h \ell}^{\ell h}+\alpha_{h \ell}^{\ell \ell}+\beta_{h \ell}\right) u^{\prime}\left(w_{h \ell}^{00}\right)-\gamma \lambda_{h \ell}\right] \\
\quad-\left[\left(1-p_{h}\right)^{2} \alpha_{h h}^{h \ell}+\left(1-p_{h}\right)\left(1-p_{\ell}\right) \alpha_{\ell h}^{h \ell}\right] u^{\prime}\left(w_{h \ell}^{00}\right)+\delta_{h \ell}^{00 / 10}-\eta_{h \ell}^{00 / 01}=0 \\
\frac{\partial \mathcal{L}}{\partial w_{h \ell}^{01}}=p_{\ell}\left(1-p_{h}\right)\left[\left(\alpha_{h \ell}^{\ell h}+\alpha_{h \ell}^{\ell \ell}+\beta_{h \ell}\right) u^{\prime}\left(w_{h \ell}^{01}\right)-\gamma \lambda_{h \ell}\right] \\
\quad-\left[p_{h}\left(1-p_{h}\right) \alpha_{h h}^{h \ell}+p_{h}\left(1-p_{\ell}\right) \alpha_{\ell h}^{h \ell}\right] u^{\prime}\left(w_{h \ell}^{01}\right)+\delta_{h \ell}^{01 / 11}+\eta_{h \ell}^{00 / 01}=0 \\
\frac{\partial \mathcal{L}}{\partial w_{h \ell}^{11}}=p_{h} p_{\ell}\left[\left(\alpha_{h \ell}^{\ell h}+\alpha_{h \ell}^{\ell \ell}+\beta_{h \ell}\right) u^{\prime}\left(w_{h \ell}^{11}\right)-\gamma \lambda_{h \ell}\right] \\
\frac{\partial \mathcal{L}}{\partial w_{h \ell}^{10}}=p_{h}\left(1-p_{\ell}\right)\left[\left(\alpha_{h \ell}^{\ell h}+\alpha_{h \ell}^{\ell \ell}+\beta_{h \ell}\right) u^{\prime}\left(w_{h \ell}^{10}\right)-\gamma \lambda_{h \ell}\right] \\
-\left[p_{h}\left(1-p_{h}\right) \alpha_{h h}^{h \ell}+p_{\ell}\left(1-p_{\ell h}^{h \ell}\right) \alpha_{\ell h}^{h \ell}\right] u^{\prime}\left(w_{h \ell}^{11}\right)-\delta_{h \ell}^{01 / 11}+\eta_{h \ell}^{10 / 11}=0 \\
\\
\quad-\delta_{h \ell}^{00 / 10}-\eta_{h \ell}^{10 / 11}=0
\end{gathered}
$$

\footnotetext{
${ }^{22}$ This may sound obvious, since it can be expected that relaxing the $B C$ constraint allows us to reach a Pareto-dominating allocation. However, taking the incentive compatibility constraints into account requires special attention.
} 
with symmetric conditions for $w_{\ell h}$, and

$$
\begin{aligned}
\frac{\partial \mathcal{L}}{\partial w_{\ell \ell}^{00}}= & \left(1-p_{\ell}\right)^{2}\left[u^{\prime}\left(w_{\ell \ell}^{00}\right)-\gamma \lambda_{\ell \ell}\right] \\
& -\left[\left(1-p_{h}\right)\left(1-p_{\ell}\right)\left(\alpha_{h \ell}^{\ell \ell}+\alpha_{\ell h}^{\ell \ell}\right)+\left(1-p_{h}\right)^{2} \alpha_{h h}^{\ell \ell}\right] u^{\prime}\left(w_{\ell \ell}^{00}\right) \\
& -\eta_{\ell \ell}^{00 / 01}-\eta_{\ell \ell}^{00 / 10}=0, \\
\frac{\partial \mathcal{L}}{\partial w_{\ell \ell}^{01}}= & p_{\ell}\left(1-p_{\ell}\right)\left[u^{\prime}\left(w_{\ell \ell}^{01}\right)-\gamma \lambda_{\ell \ell}\right] \\
& -\left[p_{\ell}\left(1-p_{h}\right) \alpha_{h \ell}^{\ell \ell}+p_{h}\left(1-p_{\ell}\right) \alpha_{\ell h}^{\ell \ell}+p_{h}\left(1-p_{h}\right) \alpha_{h h}^{\ell \ell}\right] u^{\prime}\left(w_{\ell \ell}^{01}\right) \\
& +\eta_{\ell \ell}^{00 / 01}-\eta_{\ell \ell}^{01 / 11}=0, \\
\frac{\partial \mathcal{L}}{\partial w_{\ell \ell}^{10}}= & p_{\ell}\left(1-p_{\ell}\right)\left[u^{\prime}\left(w_{\ell \ell}^{10}\right)-\gamma \lambda_{\ell \ell}\right] \\
& -\left[p_{h}\left(1-p_{\ell}\right) \alpha_{h \ell}^{\ell \ell}+p_{\ell}\left(1-p_{h}\right) \alpha_{\ell h}^{\ell \ell}+p_{h}\left(1-p_{h}\right) \alpha_{h h}^{\ell \ell}\right] u^{\prime}\left(w_{\ell \ell}^{10}\right) \\
& +\eta_{\ell \ell}^{00 / 10}-\eta_{\ell \ell}^{10 / 11}=0, \\
\frac{\partial \mathcal{L}}{\partial w_{\ell \ell}^{11}}= & p_{\ell}^{2}\left[\left(u^{\prime}\left(w_{\ell \ell}^{11}\right)-\gamma \lambda_{\ell \ell}\right]\right. \\
& -\left[p_{h} p_{\ell}\left(\alpha_{h \ell}^{\ell \ell}+\alpha_{\ell h}^{\ell \ell}\right)+p_{h}^{2} \alpha_{h h}^{\ell \ell}\right] u^{\prime}\left(w_{\ell}^{11}\right) \\
& +\eta_{\ell \ell}^{01 / 11}+\eta_{\ell \ell}^{10 / 11}=0 .
\end{aligned}
$$

Let us characterize $w_{h h}, w_{h \ell}, w_{\ell h}$ and $w_{\ell \ell}$ successively.

\section{1: Characterization of $w_{h h}$}

Using $\gamma>0$ and (22)-(25) gives $\alpha_{h h}^{h \ell}+\alpha_{h h}^{\ell h}+\alpha_{h h}^{\ell \ell}+\beta_{h h}>0$. Using $u^{\prime \prime}<0$ gives (10).

2: Characterization of $w_{h \ell}$ and $w_{\ell h}$ 
Equations (26)-(29) may be rewritten as

$$
\begin{aligned}
& u^{\prime}\left(w_{h \ell}^{00}\right)\left[\alpha_{h \ell}^{\ell h}+\alpha_{h \ell}^{\ell \ell}+\beta_{h \ell}-\alpha_{h h}^{h \ell} \frac{1-p_{h}}{1-p_{\ell}}-\alpha_{\ell h}^{h \ell}\right]=\gamma \lambda_{h \ell}-\frac{\delta_{h \ell}^{00 / 10}-\eta_{h \ell}^{00 / 01}}{\left(1-p_{h}\right)\left(1-p_{\ell}\right)}, \\
& u^{\prime}\left(w_{h \ell}^{01}\right)\left[\alpha_{h \ell}^{\ell h}+\alpha_{h \ell}^{\ell \ell}+\beta_{h \ell}-\alpha_{h h}^{h \ell} \frac{p_{h}}{p_{\ell}}-\alpha_{\ell h}^{h \ell} \frac{p_{h}\left(1-p_{\ell}\right)}{p_{\ell}\left(1-p_{h}\right)}\right]=\gamma \lambda_{h \ell}-\frac{\delta_{h \ell}^{01 / 11}+\eta_{h \ell}^{00 / 01}}{p_{\ell}\left(1-p_{h}\right)}, \\
& u^{\prime}\left(w_{h \ell}^{10}\right)\left[\alpha_{h \ell}^{\ell h}+\alpha_{h \ell}^{\ell \ell}+\beta_{h \ell}-\alpha_{h h}^{h \ell} \frac{1-p_{h}}{1-p_{\ell}}-\alpha_{\ell h}^{h \ell} \frac{p_{\ell}\left(1-p_{h}\right)}{p_{h}\left(1-p_{\ell}\right)}\right]=\gamma \lambda_{h \ell}+\frac{\delta_{h \ell}^{00 / 10}+\eta_{h \ell}^{10 / 11}}{p_{h}\left(1-p_{\ell}\right)} \\
& u^{\prime}\left(w_{h \ell}^{11}\right)\left[\alpha_{h \ell}^{\ell h}+\alpha_{h \ell}^{\ell \ell}+\beta_{h \ell}-\alpha_{h h}^{h \ell} \frac{p_{h}}{p_{\ell}}-\alpha_{\ell h}^{h \ell}\right]=\gamma \lambda_{h \ell}+\frac{\delta_{h \ell}^{01 / 11}-\eta_{h \ell}^{10 / 11}}{p_{h} p_{\ell}} .
\end{aligned}
$$

Let us consider 4 possible cases according to whether $N N I_{h \ell}^{00 / 01}$ and $N N I_{h \ell}^{10 / 11}$ are tight or not.

Case 1: $w_{h \ell}^{01}>w_{h \ell}^{00}-L$ and $w_{h \ell}^{11}>w_{h \ell}^{10}-L$, with $\eta_{h \ell}^{00 / 01}=\eta_{h \ell}^{10 / 11}=0$.

We have $w_{h \ell}^{10} \leq w_{h \ell}^{00}$ from $N O I_{h \ell}^{00 / 10}$. Suppose $w_{h \ell}^{10}<w_{h \ell}^{00}$, and thus $\delta_{h \ell}^{00 / 10}=$ 0 . Using $u^{\prime \prime}<0$ gives $u^{\prime}\left(w_{h \ell}^{10}\right)>u^{\prime}\left(w_{h \ell}^{00}\right)$. Then, $p_{\ell}<p_{h}$ and non-negativity conditions on multipliers imply that the LHS of (34) is smaller than the LHS of (36), which contradicts the fact that the RHS of (34) and (36) are equal to $\gamma \lambda_{h \ell}$. Thus, we have $w_{h \ell}^{10}=w_{h \ell}^{00}$. Similarly, we have $w_{h \ell}^{11} \leq w_{h \ell}^{01}$ from $N O I_{h \ell}^{01 / 11}$. Suppose $w_{h \ell}^{11}<w_{h \ell}^{01}$, with $\delta_{h \ell}^{01 / 11}=0$. By a similar argument, (35) and (37) lead us to a contradiction, and thus we have $w_{h \ell}^{11}=w_{h \ell}^{01}$. Suppose $w_{h \ell}^{11} \geq w_{h \ell}^{00}$, and thus

$$
w_{h \ell}^{11}=w_{h \ell}^{01} \geq w_{h \ell}^{10}=w_{h \ell}^{00}
$$

We have

$$
u_{h h}^{*}=U_{h h}\left(w_{h h}\right) \geq U_{h h}\left(w_{h \ell}\right) \geq U_{h \ell}\left(w_{h \ell}\right)=u_{h \ell}^{*},
$$

where the first and second inequalities follow from $I R_{h h}$ and $p_{\ell}<p_{h}$, respectively. This contradicts (9). Hence, we have $w_{h \ell}^{11}<w_{h \ell}^{00}$, and thus (11) holds in that case.

Case 2: $w_{h \ell}^{01}=w_{h \ell}^{00}-L$ and $w_{h \ell}^{11}=w_{h \ell}^{10}-L$, with $\eta_{h \ell}^{00 / 01} \geq 0, \eta_{h \ell}^{10 / 11} \geq 0$. We have

$$
L=w_{h \ell}^{00}-w_{h \ell}^{01}=w_{h \ell}^{10}-w_{h \ell}^{11} .
$$


Suppose that $N O I_{h \ell}^{00 / 10}$ is not binding, and thus that $w_{h \ell}^{10}<w_{h \ell}^{00}$. We deduce that $w_{h \ell}^{01}>w_{h \ell}^{11}$, i.e. $N O I_{h \ell}^{01 / 11}$ is also not binding. We thus have $\delta_{h \ell}^{00 / 10}=$ $\delta_{h \ell}^{01 / 11}=0$ and $u^{\prime}\left(w_{h \ell}^{00}\right)<u^{\prime}\left(w_{h \ell}^{10}\right)$. By an argument similar to the one used in case $1,(34)$ and (36) show that

$$
\frac{\eta_{h \ell}^{00 / 01}}{1-p_{h}}<\frac{\eta_{h \ell}^{10 / 11}}{p_{h}},
$$

and (35) and (37) show that

$$
\frac{\eta_{h \ell}^{00 / 01}}{\left(1-p_{h}\right)}>\frac{\eta_{h \ell}^{10 / 11}}{p_{h}}
$$

hence a contradiction. We thus have $w_{h \ell}^{10}=w_{h \ell}^{00}$ and $w_{h \ell}^{01}=w_{h \ell}^{11}$.

In these first two cases, (11) is satisfied and condition (2) shows that risk-specific policies are optimal for type $h \ell$ households with full coverage for $r=1$ and either partial coverage for $r=2$ (case 1) or zero coverage for $r=2$ (case 2).

It remains to show that the two remaining cases do not occur at an optimal solution to the relaxed problem.

Case 3: $w_{h \ell}^{01}=w_{h \ell}^{00}-L$ and $w_{h \ell}^{11}>w_{h \ell}^{10}-L$, with $\eta_{h \ell}^{00 / 01} \geq 0, \eta_{h \ell}^{10 / 11}=0$.

Using $w_{h \ell}^{01} \geq w_{h \ell}^{11}$ from $N O I_{h \ell}^{01 / 11}$ gives $w_{h \ell}^{00}>w_{h \ell}^{10}$, and thus $\delta_{h \ell}^{00 / 10}=0$ because $N O I_{h \ell}^{00 / 10}$ is not binding. (34),(36) and $p_{h}>p_{\ell}$ imply $w_{h \ell}^{00} \leq w_{h \ell}^{10}$, hence a contradiction.

Case 4: $w_{h \ell}^{01}>w_{h \ell}^{00}-L$ and $w_{h \ell}^{11}=w_{h \ell}^{10}-L$, with $\eta_{h \ell}^{00 / 01}=0, \eta_{h \ell}^{10 / 11} \geq 0$. Using $w_{h \ell}^{00} \geq w_{h \ell}^{10}$ from $N O I_{h \ell}^{00 / 10}$ gives $w_{h \ell}^{01}>w_{h \ell}^{11}$, and thus $\delta_{h \ell}^{01 / 11}=0$ because $N O I_{h \ell}^{01 / 11}$ is not binding. (35),(37) and $p_{h}>p_{\ell}$ imply $w_{h \ell}^{01} \leq w_{h \ell}^{11}$, hence a contradiction.

A symmetrical characterization is obtained for $w_{\ell h}$.

\section{3: Characterization of $w_{\ell \ell}$}

We have

$$
\begin{aligned}
& w_{\ell \ell}^{00} \geq \min \left\{w_{\ell \ell}^{01}, w_{\ell \ell}^{10}\right\} \\
& w_{\ell \ell}^{11} \leq \max \left\{w_{\ell \ell}^{01}, w_{\ell \ell}^{10}\right\}
\end{aligned}
$$


from the nonover-insurance constraints. If both these inequalities were binding, then we would have

$$
w_{\ell \ell}^{00}=w_{\ell \ell}^{01}=w_{\ell \ell}^{10}=w_{\ell \ell}^{11},
$$

or, in other words, there would be full coverage for type $\ell \ell$ households. Using $I R_{h h}, I C_{h h}^{\ell \ell}$ and the fact that the expected utility of type $\ell \ell$ household is larger or equal to $u_{\ell \ell}^{*}$ in the relaxed problem, would allow us to write

$$
u_{h h}^{*}=U_{h h}\left(w_{h h}\right) \geq U_{h h}\left(w_{\ell \ell}\right)=U_{\ell \ell}\left(w_{\ell \ell}\right) \geq u_{\ell \ell}^{*},
$$

which contradicts $(9)$. Thus, we have $w_{\ell \ell}^{00}>\min \left\{w_{\ell \ell}^{01}, w_{\ell \ell}^{10}\right\}$ and/or $w_{\ell \ell}^{11}<$ $\max \left\{w_{\ell \ell}^{01}, w_{\ell \ell}^{10}\right\}$.

\section{Stage 2}

Let us show that the optimal solution of the relaxed problem satisfies the constraints that were ignored at stage 1.

1: Constraints $I C_{\ell \ell}^{h \ell}$ and $I C_{\ell \ell}^{\ell h}$.

If $I C_{\ell \ell}^{h \ell}$ were not satisfied, then we would have $U_{\ell \ell}\left(w_{\ell \ell}\right)<U_{\ell \ell}\left(w_{h \ell}\right)$. We have

$$
U_{\ell \ell}\left(w_{h \ell}\right)=\left(1-p_{\ell}\right) u\left(w_{h \ell}^{00}\right)+p_{\ell} u\left(w_{h \ell}^{11}\right)=U_{h \ell}\left(w_{h \ell}\right)=u_{h \ell}^{*},
$$

from (11), and thus $U_{\ell \ell}\left(w_{\ell \ell}\right)<u_{h \ell}^{*}$. At the optimal solution of the relaxed problem, the expected utility of type $\ell \ell$ households is at least equal to $u_{\ell \ell}^{*}$, which is its value at the optimal solution of the complete problem. Thus, we have $u_{\ell \ell}^{*} \leq U_{\ell \ell}\left(w_{\ell \ell}\right)$, which implies $u_{\ell \ell}^{*}<u_{h \ell}^{*}$, which contradicts (9). The same argument applies for $I C_{\ell \ell}^{\ell h}$.

2: Constraints $I C_{h \ell}^{h h}$ and $I C_{\ell h}^{h h}$.

If $I C_{h \ell}^{h h}$ were not satisfied, then we would have $U_{h \ell}\left(w_{h \ell}\right)<U_{h \ell}\left(w_{h h}\right)$. Furthermore, (10) gives $U_{h \ell}\left(w_{h h}\right)=U_{h h}\left(w_{h h}\right)=u_{h h}^{*}$. Using $u_{h \ell}^{*}=U_{h \ell}\left(w_{h \ell}\right)$ would then yield $u_{h \ell}^{*}<u_{h h}^{*}$, which contradicts (9). The same argument holds for $I C_{\ell h}^{h h}$.

3: Constraint $I C_{\ell \ell}^{h h}$.

We may write

$$
\begin{aligned}
U_{\ell \ell}\left(w_{\ell \ell}\right) & \geq\left(1-p_{\ell}\right) u\left(w_{h \ell}^{00}\right)+p_{\ell} u\left(w_{h \ell}^{11}\right) \\
& >\left(1-p_{h}\right) u\left(w_{h \ell}^{00}\right)+p_{h} u\left(w_{h \ell}^{11}\right) \\
& \geq U_{h h}\left(w_{h h}\right) \\
& =U_{\ell \ell}\left(w_{h h}\right)
\end{aligned}
$$


where (38),(39),(40) and (41) result from $I C_{\ell \ell}^{h \ell}$, from $p_{h}>p_{\ell}$ and (11), from $I C_{h \ell}^{h h}$ and from (10), respectively. Thus, $I C_{\ell \ell}^{h h}$ is satisfied.

4: The no-overinsurance and non-negative indemnity constraints ignored at stage 1 are obviously satisfied at an optimal solution of the relaxed problem.

\section{Lemma 1}

The Lagrange multiplier $\gamma$ associated with the $B C$ constraint in the relaxed problem is strictly positive.

\section{Proof of Lemma 1:}

The proof consists in showing that an exogenous increase in the r.h.s. of the $B C$ constraint yields feasible allocations that dominate the optimal solution to the relaxed problem. To do so, consider an optimal solution $w=\left(w_{h h}, w_{h \ell}, w_{\ell h}, w_{\ell \ell}\right)$ to the relaxed problem and write the r.h.s. of $B C$ as $\bar{w}+m$, with $m \geq 0$. Let $u_{\ell \ell}(m)$ be the type $\ell \ell$ expected utility at an optimal solution of the relaxed problem, parametrized by $m$. We have

$$
{\frac{\partial u_{\ell \ell}}{\partial m}}_{\mid m=0}=\gamma \geq 0
$$

from the envelope theorem.

Let $\varepsilon>0$ and $w^{\prime}=\left(w_{h h}^{\prime}, w_{h \ell}^{\prime}, w_{\ell h}^{\prime}, w_{\ell \ell}^{\prime}\right)$ such that $w_{h h}^{x y \prime}=w_{h h}^{x y}+\varepsilon$ if $x, y \in\{\in 0,1\}$ and $w_{i j}^{\prime}=w_{i j}$ if $(i, j) \neq(h, h)$. Thus, $I_{h h}^{h \ell}, I_{h h}^{\ell h}$ and $I_{h h}^{\ell \ell}$ are satisfied and non-binding for $w^{\prime}$. For $\varepsilon^{\prime}>0$ small enough, there exists $w^{\prime \prime}=\left(w_{h h}^{\prime \prime}, w_{h \ell}^{\prime \prime}, w_{\ell h}^{\prime \prime}, w_{\ell \ell}^{\prime \prime}\right)$ such that $w_{h \ell}^{x y \prime \prime}>w_{h \ell}^{x y \prime}$ and $w_{\ell h}^{x y \prime \prime}>w_{\ell h}^{x y \prime}$ for all $x, y \in\{\in 0,1\}$ and $w_{h h}^{\prime \prime}=w_{h h}^{\prime}, w_{\ell \ell}^{\prime \prime}=w_{\ell \ell}^{\prime}$, such that

$$
C_{h \ell}\left(w_{h \ell}^{\prime \prime}\right)=C_{h \ell}\left(w_{h \ell}^{\prime}\right)+\varepsilon^{\prime}, C_{\ell h}\left(w_{\ell h}^{\prime \prime}\right)=C_{\ell h}\left(w_{\ell h}^{\prime}\right)+\varepsilon^{\prime},
$$

and such that the allocation $w^{\prime \prime}$ satisfies $I_{h h}^{h \ell}, I_{h h}^{\ell h}, I_{h h}^{\ell \ell}, I_{h \ell}^{\ell h}, I_{h \ell}^{\ell \ell}, I_{\ell h}^{h \ell}, I_{\ell h}^{\ell \ell}$, these constraints being non-binding, as well as $N O I_{h \ell}^{00 / 10}, N O I_{h \ell}^{01 / 11}, N O I_{\ell h}^{00 / 01}, N O I_{\ell h}^{10 / 11}$, $N O I_{\ell \ell}^{00 / 01}, N O I_{\ell \ell}^{00 / 10}, N O I_{\ell \ell}^{01 / 11}, N O I_{\ell \ell}^{10 / 11}{ }^{23}$ Since $\varepsilon^{\prime}>0$ exists when $\varepsilon>0$, we may write $\varepsilon^{\prime}=\varphi_{1}(\varepsilon)$ with $\varphi_{1}(0)=0$ and $\varphi_{1}^{\prime}>0$. Finally, let $\varepsilon^{\prime \prime}>0$ and $w^{\prime \prime \prime}=\left(w_{h h}^{\prime \prime \prime}, w_{h \ell}^{\prime \prime \prime}, w_{\ell h}^{\prime \prime \prime}, w_{\ell \ell}^{\prime \prime \prime}\right)$ such that $w_{\ell \ell}^{x y \prime \prime \prime}=w_{\ell \ell}^{x y \prime \prime}+\varepsilon^{\prime \prime}$ if $x, y \in\{\in 0,1\}$ and $w_{i j}^{\prime \prime \prime}=w_{i j}^{\prime \prime}$ if $(i, j) \neq(\ell, \ell)$. $w^{\prime \prime \prime}$ satisfies all the constraints of the relaxed

\footnotetext{
${ }^{23}$ Intuitively, $w_{h \ell}^{\prime \prime}$ and $w_{\ell h}^{\prime \prime}$ are chosen in such a way that the incentive compatibility constraints of types $h h, h \ell$ and $\ell h$ are still satisfied and the expected utility of types $h \ell$ and $\ell h$ is increasing.
} 
problem except $B C$ if $\varepsilon^{\prime \prime}$ is small enough. Since $\varepsilon^{\prime \prime}>0$ exists when $\varepsilon^{\prime}>0$, we may write $\varepsilon^{\prime \prime}=\varphi_{2}\left(\varepsilon^{\prime}\right)$ with $\varphi_{2}(0)=0$ and $\varphi_{2}^{\prime}>0$.

The variations in the l.h.s. of $B C$ and in the expected utility of type $\ell \ell$ households induced by the change from $w$ to $w^{\prime \prime \prime}$ are functions of $\varepsilon$ that may be written as

$$
\begin{aligned}
\Delta_{B C}(\varepsilon)= & \sum_{i, j \in\{h, \ell\}} \lambda_{i j}\left[C_{i j}\left(w_{i j}^{\prime \prime \prime}\right)-C_{i j}\left(w_{i j}\right)\right]=\lambda_{h h}+\left(\lambda_{h \ell}+\lambda_{\ell h}\right) \varphi_{1}(\varepsilon)+\lambda_{\ell \ell} \varphi_{2}\left(\varphi_{1}(\varepsilon)\right), \\
\Delta_{u_{\ell \ell}}(\varepsilon)= & \left(1-p_{\ell}\right)^{2}\left[u\left(w_{\ell \ell}^{00}+\varphi_{2}\left(\varphi_{1}(\varepsilon)\right)-u\left(w_{\ell \ell}^{00}\right)\right]\right. \\
& +p_{\ell}\left(1-p_{\ell}\right)\left[u \left(w_{\ell \ell}^{01}+\varphi_{2}\left(\varphi_{1}(\varepsilon)\right)-u\left(w_{\ell \ell}^{01}\right)+u\left(w_{\ell \ell}^{10}+\varphi_{2}\left(\varphi_{1}(\varepsilon)\right)-u\left(w_{\ell \ell}^{10}\right)\right]\right.\right. \\
& +p_{\ell}^{2}\left[u\left(w_{\ell \ell}^{11}+\varphi_{2}\left(\varphi_{1}(\varepsilon)\right)-u\left(w_{\ell \ell}^{11}\right)\right],\right.
\end{aligned}
$$

respectively, with $\Delta_{B C}^{\prime}(0)>0, \Delta_{u_{\ell}}^{\prime}(0)>0$.

Since $w^{\prime \prime \prime}$ is only one of the incentive compatible allocations that induce the same increase in cost, we deduce

$$
\gamma=\frac{\partial u_{\ell \ell}}{\partial m} \geq \frac{\Delta_{u_{\ell \ell}}^{\prime}(0)}{\Delta_{B C}^{\prime}(0)}>0
$$

\section{Proof of Proposition 3}

The first part of the proposition was established in section 3. It remains to show that $w_{\ell \ell}^{00}+w_{\ell \ell}^{11}>w_{\ell \ell}^{10}+w_{\ell \ell}^{01}$ when $u^{\prime \prime \prime}>0$ and $\lambda_{h h}$ is small enough. To give a precise meaning to this latter assumption, we consider a sequence of optimal allocation problems indexed by $n \in \mathbb{N}$, where the fraction of type $i j$ households and their expected utility are denoted by $\lambda_{i j}^{n}$ and $u_{i j}^{* n}$, rexpectively, for $i, j \in\{h, \ell\}$, with $\lambda_{h h}^{n}+\lambda_{h \ell}^{n}+\lambda_{\ell h}^{n}+\lambda_{\ell \ell}^{n}=1$ for all $n$. Let $w_{i j}^{n}=$ $\left(w_{i j}^{00 n}, w_{i j}^{01 n}, w_{i j}^{10 n}, w_{i j}^{11 n}\right)$ be the state-contingent wealth of type $i j$ households, at a second-best Pareto optimal allocation corresponding to index $n$. Assume that $\lambda_{i j}^{n} \rightarrow \lambda_{i j}^{\infty}, u_{i j}^{* n} \rightarrow u_{i j}^{* \infty}, w_{i j}^{n} \rightarrow w_{i j}^{\infty}=\left(w_{i j}^{00 \infty}, w_{i j}^{01 \infty}, w_{i j}^{10 \infty}, w_{i j}^{11 \infty}\right)$ when $n \rightarrow \infty$, with $\lambda_{h h}^{\infty}=0$, and (9) holds for all $n$. We will show that

$$
w_{\ell \ell}^{00 n}+w_{\ell \ell}^{11 n}>w_{\ell \ell}^{10 n}+w_{\ell \ell}^{01 n}
$$

for $n$ large enough.

Observe first that, if we replace $I R_{h h}^{n}$ by an inequality $U_{h h}\left(w_{h h}\right) \geq u_{h h}^{*}$, with non-negative multiplier $\beta_{h h}$ in the optimization problem that characterizes a second-best Pareto-optimal allocation, then this inequality will be binding at an optimal solution to this problem, for otherwise there would 
be a contradiction with second-best Pareto-optimality. ${ }^{24}$ Furthermore, we know from step 2 in the proof of Proposition 2 that $I C_{h \ell}^{h h n}, I C_{\ell h}^{h h n}$ and $I C_{\ell \ell}^{h h n}$ are not binding at such an optimal solution when (9) holds. Consequently, conditions (22)-(25) characterize all second-best Pareto-optimal allocations in our sequence, with non-negative multipliers $\left\{\alpha_{h h}^{h \ell n}, \alpha_{h h}^{\ell h n}, \alpha_{h h}^{\ell \ell n}, \beta_{h h}^{n}\right\}_{n \in \mathbb{N}}$ that converge to $\left(\alpha_{h h}^{h \ell \infty}, \alpha_{h h}^{\ell h \infty}, \alpha_{h h}^{\ell \ell \infty}, \beta_{h h}^{\infty}\right)$ when $n \rightarrow \infty .^{25}$ Since (10) holds for all terms of the sequence, we have $w_{h h}^{00 \infty}=w_{h h}^{01 \infty}=w_{h h}^{10 \infty}=w_{h h}^{11 \infty}=u^{-1}\left(u_{h h}^{* \infty}\right)$ and (22)-(25) give

$$
\left(\alpha_{h h}^{h \ell \infty}+\alpha_{h h}^{\ell h \infty}+\alpha_{h h}^{\ell \ell \infty}+\beta_{h h}^{\infty}\right) u^{\prime}\left(u^{-1}\left(u_{h h}^{\infty}\right)\right)=\gamma \lambda_{h h}^{\infty}=0,
$$

which implies $\alpha_{h h}^{h \ell \infty}=\alpha_{h h}^{\ell h \infty}=\alpha_{h h}^{\ell \ell \infty}=\beta_{h h}^{\infty}=0$.

By using similar notations for multipliers $\eta_{\ell \ell}^{00 / 01 n}, \ldots$, and by denoting

$$
\begin{aligned}
z^{00 n} & =\left(\alpha_{h \ell}^{\ell \ell n}+\alpha_{\ell h}^{\ell \ell n}\right) \frac{1-p_{h}}{1-p_{\ell}}+\alpha_{h h}^{\ell \ell n} \frac{\left(1-p_{h}\right)^{2}}{\left(1-p_{\ell}\right)^{2}}, \\
z^{01 n} & =\alpha_{h \ell}^{\ell \ell n} \frac{1-p_{h}}{1-p_{\ell}}+\alpha_{\ell h}^{\ell \ell n} \frac{p_{h}}{p_{\ell}}+\alpha_{h h}^{\ell \ell n} \frac{p_{h}\left(1-p_{h}\right)}{p_{\ell}\left(1-p_{\ell}\right)}, \\
z^{10 n} & =\alpha_{h \ell}^{\ell \ell n} \frac{p_{h}}{p_{\ell}}+\alpha_{\ell h}^{\ell \ell n} \frac{1-p_{h}}{1-p_{\ell}}+\alpha_{h h}^{\ell \ell n} \frac{p_{h}\left(1-p_{h}\right)}{p_{\ell}\left(1-p_{\ell}\right)}, \\
z^{11 n} & =\left(\alpha_{h \ell}^{\ell \ell n}+\alpha_{\ell h}^{\ell \ell n}\right) \frac{p_{h}}{p_{\ell}}+\alpha_{h h}^{\ell \ell n} \frac{p_{h}^{2}}{p_{\ell}^{2}},
\end{aligned}
$$

equations (30)-(33) may be more compacty rewritten as

$$
\begin{aligned}
& u^{\prime}\left(w_{\ell \ell}^{00 n}\right)\left[1-z^{00 n}\right]=\gamma \lambda_{\ell \ell}+\frac{\eta_{\ell \ell}^{00 / 01 n}+\eta_{\ell \ell}^{00 / 10 n}}{\left(1-p_{\ell}\right)^{2}}, \\
& u^{\prime}\left(w_{\ell \ell}^{01 n}\right)\left[1-z^{01 n}\right]=\gamma \lambda_{\ell \ell}-\frac{\eta_{\ell \ell}^{00 / 01 n}-\eta_{\ell \ell}^{01 / 11 n}}{p_{\ell}\left(1-p_{\ell}\right)}, \\
& u^{\prime}\left(w_{\ell \ell}^{10 n}\right)\left[1-z^{10 n}\right]=\gamma \lambda_{\ell \ell}-\frac{\eta_{\ell \ell}^{00 / 10 n}-\eta_{\ell \ell}^{10 / 11 n}}{p_{\ell}\left(1-p_{\ell}\right)}, \\
& u^{\prime}\left(w_{\ell \ell}^{11 n}\right)\left[1-z^{11 n}\right]=\gamma \lambda_{\ell \ell}-\frac{\eta_{\ell \ell}^{01 / 11 n}+\eta_{\ell \ell}^{10 / 11 n}}{p_{\ell}^{2}},
\end{aligned}
$$

\footnotetext{
${ }^{24}$ We add the subscript $n$ to refer to the variables or constraints of the problem indexed by $n$.

${ }^{25}$ In the proof of Proposition 2, there was no sign condition on $\beta_{h h}$ because $I R_{h h}$ was written as an equality constraint.
} 
Consider the various possible cases.

Case 1: $N N I_{\ell \ell}^{00 / 01 n}, N N I_{\ell \ell}^{00 / 10 n}, N N I_{\ell \ell}^{01 / 11 n}, N N I_{\ell \ell}^{10 / 11 n}$ are not binding, ${ }^{26}$ which gives $\eta_{\ell \ell}^{00 / 01 n}=\eta_{\ell \ell}^{00 / 10 n}=\eta_{\ell \ell}^{01 / 11 n}=\eta_{\ell \ell}^{10 / 11 n}=0$.

Let $\Phi(z)=u^{\prime-1}\left(\gamma \lambda_{\ell \ell} / 1-z\right)$, with $0 \leq z<1$. $u^{\prime \prime}<0$ gives $\Phi^{\prime}<0$, with $\Phi^{\prime \prime}>0$ if $u^{\prime \prime \prime}>0$. Equations (43)-(46) give $w_{\ell \ell}^{x y n}=\Phi\left(z^{x y n}\right)$ for $(x, y) \in\{0,1\}$.

Let $\left(z^{00 n}, z^{01 n}, z^{10 n}, z^{11 n}\right) \rightarrow\left(z^{00 \infty}, z^{01 \infty}, z^{10 \infty}, z^{11 \infty}\right)$ when $n \rightarrow \infty$. Using $\alpha_{h h}^{\ell \ell \infty}=0$ yields

$$
z^{00 \infty}+z^{11 \infty}=z^{01 \infty}+z^{10 \infty} .
$$

Furthermore, $z^{11 \infty} \leq \min \left\{z^{01 \infty}, z^{10 \infty}\right\} \leq \max \left\{z^{01 \infty}, z^{10 \infty}\right\} \leq z^{00 \infty}$, with at least one inequality not tight, shows that lottery $\left(z^{00 \infty}, 1 / 2 ; z^{11 \infty}, 1 / 2\right)$ is obtained from lottery $\left(z^{01 \infty}, 1 / 2 ; z^{10 \infty}, 1 / 2\right)$ by a mean-preserving spread. Using $\Phi^{\prime \prime}>0$ gives

$$
\begin{aligned}
w_{\ell \ell}^{00 \infty}+w_{\ell \ell}^{11 \infty} & =\Phi\left(z^{00 \infty}\right)+\Phi\left(z^{11 \infty}\right) \\
& >\Phi\left(z^{01 \infty}\right)+\Phi\left(z^{10 \infty}\right) \\
& =w_{\ell \ell}^{01 \infty}+w_{\ell \ell}^{10 \infty} .
\end{aligned}
$$

Since $w_{\ell \ell}^{n} \rightarrow w_{\ell \ell}^{\infty}$ when $n \rightarrow \infty$, we deduce that (42) holds for $n$ large enough.

Case 2: $N N I_{\ell \ell}^{00 / 01 n}$ and/or $N N I_{\ell \ell}^{00 / 10 n}$ is binding.

Assume that $N N I_{\ell \ell}^{00 / 01 n}$ is binding, which gives $w_{\ell \ell}^{00 n}-w_{\ell \ell}^{01 n}=L$. We also have $w_{\ell \ell}^{11 n}-w_{\ell \ell}^{10 n} \geq-L$ from $N N I_{\ell \ell}^{10 / 11 n}$. We deduce

$$
w_{\ell \ell}^{11 n}-w_{\ell \ell}^{10 n} \geq-L=w_{\ell \ell}^{01 n}-w_{\ell \ell}^{00 n},
$$

which gives (42). Symmetrically if $N N I_{\ell \ell}^{00 / 10 n}$ is binding.

Case 3: $N N I_{\ell \ell}^{00 / 01 n}$ and $N N I_{\ell \ell}^{00 / 10 n}$ are not binding and $N N I_{\ell \ell}^{01 / 11 n}$ and/or $N N I_{\ell \ell}^{10 / 11 n}$ is binding. Using $\eta_{\ell \ell}^{00 / 01 n}=\eta_{\ell \ell}^{00 / 10 n}=0, \eta_{\ell \ell}^{01 / 11 n}, \eta_{\ell \ell}^{10 / 11 n} \geq 0$ yields $w_{\ell \ell}^{00 n}=\Phi\left(z^{00 n}\right), w_{\ell \ell}^{01 n} \leq \Phi\left(z^{01 n}\right), w_{\ell \ell}^{10 n} \leq \Phi\left(z^{10 n}\right), w_{\ell \ell}^{11 n} \geq \Phi\left(z^{11 n}\right)$. As in Case 1, the no-oversinsurance constraints are satisfied, and let us consider a sequence $\left(z^{00 n}, z^{01 n}, z^{10 n}, z^{11 n}\right) \rightarrow\left(z^{00 \infty}, z^{01 \infty}, z^{10 \infty}, z^{11 \infty}\right)$ when $n \rightarrow \infty$, with

$$
z^{00 \infty}+z^{11 \infty}=z^{01 \infty}+z^{10 \infty},
$$

\footnotetext{
${ }^{26}$ We refer to the problem whose parameters are indexed by $n \in \mathbb{N}$.
} 
and $w_{\ell \ell}^{00 \infty}=\Phi\left(z^{00 \infty}\right), w_{\ell \ell}^{01 \infty} \leq \Phi\left(z^{01 \infty}\right), w_{\ell \ell}^{10 \infty} \leq \Phi\left(z^{10 \infty}\right), w_{\ell \ell}^{11 \infty} \geq \Phi\left(z^{11 \infty}\right)$. Using the previous equalities and inequalities as well as $\Phi^{\prime \prime}>0$ yields

$$
\begin{aligned}
w_{\ell \ell}^{00 \infty}+w_{\ell \ell}^{11 \infty} & \geq \Phi\left(z^{00 \infty}\right)+\Phi\left(z^{11 \infty}\right) \\
& >\Phi\left(z^{01 \infty}\right)+\Phi\left(z^{10 \infty}\right) \\
& \geq w_{\ell \ell}^{01 \infty}+w_{\ell \ell}^{10 \infty}
\end{aligned}
$$

which shows that (42) holds for $n$ large enough.

\section{Proof of Proposition 4}

The proof is in two stages.

\section{Stage 1}

As in the proof of Proposition 2, we consider the relaxed problem by deleting the same incentive constraints and with unchanged notations for Lagrange multipliers. We assume that $\sigma$ is not exagerately large so that $N N I_{i j}^{00 / 01}, \ldots$ are not binding if the corresponding constraints $N O I_{i j}^{00 / 01}, \ldots$ are binding when $\sigma=0$. Hence, with unchanged notations for multipliers, we have $\eta_{h h}^{00 / 01}=\eta_{h h}^{00 / 10}=\eta_{h h}^{01 / 11}=\eta_{h h}^{10 / 11}=\eta_{h \ell}^{00 / 10}=\eta_{h \ell}^{01 / 11}=\eta_{\ell h}^{00 / 01}=\eta_{\ell h}^{10 / 11}=0$.

The first-order optimality conditions are written as

$$
\begin{aligned}
\frac{\partial \mathcal{L}}{\partial w_{h h}^{00}}= & \left(1-p_{h}\right)^{2}\left(\alpha_{h h}^{h \ell}+\alpha_{h h}^{\ell h}+\alpha_{h h}^{\ell \ell}+\beta_{h h}\right) u^{\prime}\left(w_{h h}^{00}\right) \\
& -\gamma \lambda_{h h}\left[1-(1+\sigma) p_{h}\left(2-p_{h}\right)\right]=0, \\
\frac{\partial \mathcal{L}}{\partial w_{h h}^{01}}= & p_{h}\left(1-p_{h}\right)\left[\left(\alpha_{h h}^{h \ell}+\alpha_{h h}^{\ell h}+\alpha_{h h}^{\ell \ell}+\beta_{h h}\right) u^{\prime}\left(w_{h h}^{01}\right)\right. \\
& \left.-\gamma \lambda_{h h}(1+\sigma)\right]=0, \\
\frac{\partial \mathcal{L}}{\partial w_{h h}^{10}}= & p_{h}\left(1-p_{h}\right)\left[\left(\alpha_{h h}^{h \ell}+\alpha_{h h}^{\ell h}+\alpha_{h h}^{\ell \ell}+\beta_{h h}\right) u^{\prime}\left(w_{h h}^{10}\right)\right. \\
& \left.-\gamma \lambda_{h h}(1+\sigma)\right]=0, \\
\frac{\partial \mathcal{L}}{\partial w_{h h}^{11}}= & p_{h}^{2}\left[\left(\alpha_{h h}^{h \ell}+\alpha_{h h}^{\ell h}+\alpha_{h h}^{\ell \ell}+\beta_{h h}\right) u^{\prime}\left(w_{h h}^{11}\right)\right. \\
& \left.-\gamma \lambda_{h h}(1+\sigma)\right]=0,
\end{aligned}
$$




$$
\begin{aligned}
\frac{\partial \mathcal{L}}{\partial w_{h \ell}^{00}}= & \left(1-p_{h}\right)\left(1-p_{\ell}\right)\left[\left(\alpha_{h \ell}^{\ell h}+\alpha_{h \ell}^{\ell \ell}+\beta_{h \ell}\right) u^{\prime}\left(w_{h \ell}^{00}\right)-\gamma \lambda_{h \ell} \frac{1-(1+\sigma)\left(p_{h}+p_{\ell}-p_{h} p_{\ell}\right)}{\left(1-p_{h}\right)\left(1-p_{\ell}\right)}\right] \\
& -\left[\left(1-p_{h}\right)^{2} \alpha_{h h}^{h \ell}+\left(1-p_{h}\right)\left(1-p_{\ell}\right) \alpha_{\ell h}^{h \ell}\right] u^{\prime}\left(w_{h \ell}^{00}\right)+\delta_{h \ell}^{00 / 10}-\eta_{h \ell}^{00 / 01}=0,
\end{aligned}
$$

$$
\begin{aligned}
\frac{\partial \mathcal{L}}{\partial w_{h \ell}^{01}}= & p_{\ell}\left(1-p_{h}\right)\left[\left(\alpha_{h \ell}^{\ell h}+\alpha_{h \ell}^{\ell \ell}+\beta_{h \ell}\right) u^{\prime}\left(w_{h \ell}^{01}\right)-\gamma \lambda_{h \ell}(1+\sigma)\right] \\
& -\left[p_{h}\left(1-p_{h}\right) \alpha_{h h}^{h \ell}+p_{h}\left(1-p_{\ell}\right) \alpha_{\ell h}^{h \ell}\right] u^{\prime}\left(w_{h \ell}^{01}\right)+\delta_{h \ell}^{01 / 11}+\eta_{h \ell}^{00 / 01}=0,
\end{aligned}
$$$$
\frac{\partial \mathcal{L}}{\partial w_{h \ell}^{10}}=p_{h}\left(1-p_{\ell}\right)\left[\left(\alpha_{h \ell}^{\ell h}+\alpha_{h \ell}^{\ell \ell}+\beta_{h \ell}\right) u^{\prime}\left(w_{h \ell}^{10}\right)-\gamma \lambda_{h \ell}(1+\sigma)\right]
$$$$
-\left[p_{h}\left(1-p_{h}\right) \alpha_{h h}^{h \ell}+p_{\ell}\left(1-p_{h}\right) \alpha_{\ell h}^{h \ell}\right] u^{\prime}\left(w_{h \ell}^{10}\right)-\delta_{h \ell}^{00 / 10}-\eta_{h \ell}^{10 / 11}=0,
$$

$$
\begin{aligned}
\frac{\partial \mathcal{L}}{\partial w_{h \ell}^{11}}= & p_{h} p_{\ell}\left[\left(\alpha_{h \ell}^{\ell h}+\alpha_{h \ell}^{\ell \ell}+\beta_{h \ell}\right) u^{\prime}\left(w_{h \ell}^{11}\right)-\gamma \lambda_{h \ell}(1+\sigma)\right]=0 \\
& -\left[p_{h}^{2} \alpha_{h h}^{h \ell}+p_{\ell} p_{h} \alpha_{\ell h}^{h \ell}\right] u^{\prime}\left(w_{h \ell}^{11}\right)-\delta_{h \ell}^{01 / 11}+\eta_{h \ell}^{10 / 11}=0
\end{aligned}
$$

with symmetric conditions for $w_{\ell h}$, and 


$$
\begin{aligned}
\frac{\partial \mathcal{L}}{\partial w_{\ell \ell}^{00}}= & \left(1-p_{\ell}\right)^{2}\left[u^{\prime}\left(w_{\ell \ell}^{00}\right)-\gamma \lambda_{\ell \ell} \frac{1-(1+\sigma) p_{\ell}\left(2-p_{\ell}\right)}{\left(1-p_{\ell}\right)^{2}}\right] \\
& -\left[\left(1-p_{h}\right)\left(1-p_{\ell}\right)\left(\alpha_{h \ell}^{\ell \ell}+\alpha_{\ell h}^{\ell \ell}\right)+\left(1-p_{h}\right)^{2} \alpha_{h h}^{\ell \ell}\right] u^{\prime}\left(w_{\ell \ell}^{00}\right) \\
& -\eta_{\ell \ell}^{00 / 01}-\eta_{\ell \ell}^{00 / 10}=0, \\
\frac{\partial \mathcal{L}}{\partial w_{\ell \ell}^{01}}= & p_{\ell}\left(1-p_{\ell}\right)\left[u^{\prime}\left(w_{\ell \ell}^{01}\right)-\gamma \lambda_{\ell \ell}(1+\sigma)\right] \\
& -\left[p_{\ell}\left(1-p_{h}\right) \alpha_{h \ell}^{\ell \ell}+p_{h}\left(1-p_{\ell}\right) \alpha_{\ell h}^{\ell \ell}+p_{h}\left(1-p_{h}\right) \alpha_{h h}^{\ell \ell}\right] u^{\prime}\left(w_{\ell \ell}^{01}\right) \\
& +\eta_{\ell \ell}^{00 / 01}-\eta_{\ell \ell}^{01 / 11}=0, \\
\frac{\partial \mathcal{L}}{\partial w_{\ell \ell}^{10}}= & p_{\ell}\left(1-p_{\ell}\right)\left[u^{\prime}\left(w_{\ell \ell}^{10}\right)-\gamma \lambda_{\ell \ell}(1+\sigma)\right] \\
& -\left[p_{h}\left(1-p_{\ell}\right) \alpha_{h \ell}^{\ell \ell}+p_{\ell}\left(1-p_{h}\right) \alpha_{\ell h}^{\ell \ell}+p_{h}\left(1-p_{h}\right) \alpha_{h h}^{\ell \ell}\right] u^{\prime}\left(w_{\ell \ell}^{10}\right) \\
& +\eta_{\ell \ell}^{00 / 10}-\eta_{\ell \ell}^{10 / 11}=0, \\
\frac{\partial \mathcal{L}}{\partial w_{\ell \ell}^{11}}= & p_{\ell}^{2}\left[\left(u^{\prime}\left(w_{\ell \ell}^{11}\right)-\gamma \lambda_{\ell \ell}(1+\sigma)\right]\right. \\
& -\left[p_{h} p_{\ell}\left(\alpha_{h \ell}^{\ell \ell}+\alpha_{\ell h}^{\ell \ell}\right)+p_{h}^{2} \alpha_{h h}^{\ell \ell}\right] u^{\prime}\left(w_{\ell \ell}^{11}\right) \\
& +\eta_{\ell \ell}^{01 / 11}+\eta_{\ell \ell}^{10 / 11}=0 .
\end{aligned}
$$

\section{1: Characterization of $w_{h h}$}

The same reasoning as in Lemma 1 gives $\gamma>0$, and equations (48)-(50) imply $\alpha_{h h}^{h \ell}+\alpha_{h h}^{\ell h}+\alpha_{h h}^{\ell \ell}+\beta_{h h}>0$ and $w_{h h}^{01}=w_{h \ell}^{10}=w_{h h}^{11}$, with

$$
\left(\alpha_{h h}^{h \ell}+\alpha_{h h}^{\ell h}+\alpha_{h h}^{\ell \ell}+\beta_{h h}\right) u^{\prime}\left(w_{h h}^{01}\right)=\gamma \lambda_{h h}(1+\sigma) .
$$

and equation (47) gives

$$
\begin{aligned}
\left(\alpha_{h h}^{h \ell}+\alpha_{h h}^{\ell h}+\alpha_{h h}^{\ell \ell}+\beta_{h h}\right) u^{\prime}\left(w_{h h}^{00}\right) & =\gamma \lambda_{h h} \frac{1-(1+\sigma) p_{h}\left(2-p_{h}\right)}{\left(1-p_{h}\right)^{2}} \\
& <\gamma \lambda_{h h}(1+\sigma),
\end{aligned}
$$

which implies $w_{h h}^{00}>w_{h h}^{01}$ and gives

$$
w_{h h}^{00}>w_{h h}^{10}=w_{h h}^{01}=w_{h h}^{11} .
$$

We deduce that a straight deductible is optimal for type $h h$ households, with $c_{h h}^{1}=c_{h h}^{2}=0$ and $D_{h h}=w_{h h}^{00}+w_{h h}^{11}-w_{h h}^{01}-w_{h h}^{10}<L$. 
2: Characterization of $w_{h \ell}$ and $w_{\ell h}$

Equations (51)-(54) may be rewritten as

$$
\begin{aligned}
& u^{\prime}\left(w_{h \ell}^{00}\right)\left[\alpha_{h \ell}^{\ell h}+\alpha_{h \ell}^{\ell \ell}+\beta_{h \ell}-\alpha_{h h}^{h \ell} \frac{1-p_{h}}{1-p_{\ell}}-\alpha_{\ell h}^{h \ell}\right] \\
& =\gamma \lambda_{h \ell} \frac{1-(1+\sigma)\left(p_{h}+p_{\ell}-p_{h} p_{\ell}\right)}{\left(1-p_{h}\right)\left(1-p_{\ell}\right)}-\frac{\delta_{h \ell}^{00 / 10}-\eta_{h \ell}^{00 / 01}}{\left(1-p_{h}\right)\left(1-p_{\ell}\right)}, \\
& u^{\prime}\left(w_{h \ell}^{01}\right)\left[\alpha_{h \ell}^{\ell h}+\alpha_{h \ell}^{\ell \ell}+\beta_{h \ell}-\alpha_{h h}^{h \ell} \frac{p_{h}}{p_{\ell}}-\alpha_{\ell h}^{h \ell} \frac{p_{h}\left(1-p_{\ell}\right)}{p_{\ell}\left(1-p_{h}\right)}\right] \\
& =\gamma \lambda_{h \ell}(1+\sigma)-\frac{\delta_{h \ell}^{01 / 11}+\eta_{h \ell}^{00 / 01}}{p_{\ell}\left(1-p_{h}\right)}, \\
& u^{\prime}\left(w_{h \ell}^{10}\right)\left[\alpha_{h \ell}^{\ell h}+\alpha_{h \ell}^{\ell \ell}+\beta_{h \ell}-\alpha_{h h}^{h \ell} \frac{1-p_{h}}{1-p_{\ell}}-\alpha_{\ell h}^{h \ell} \frac{p_{\ell}\left(1-p_{h}\right)}{p_{h}\left(1-p_{\ell}\right)}\right] \\
& =\gamma \lambda_{h \ell}(1+\sigma)+\frac{\delta_{h \ell}^{00 / 10}+\eta_{h \ell}^{10 / 11}}{p_{h}\left(1-p_{\ell}\right)} \\
& u^{\prime}\left(w_{h \ell}^{11}\right)\left[\alpha_{h \ell}^{\ell h}+\alpha_{h \ell}^{\ell \ell}+\beta_{h \ell}-\alpha_{h h}^{h \ell} \frac{p_{h}}{p_{\ell}}-\alpha_{\ell h}^{h \ell}\right] \\
& =\gamma \lambda_{h \ell}(1+\sigma)+\frac{\delta_{h \ell}^{01 / 11}-\eta_{h \ell}^{10 / 11}}{p_{h} p_{\ell}} .
\end{aligned}
$$

Let us consider 4 possible cases according to whether $N N I_{h \ell}^{00 / 01}$ and $N N I_{h \ell}^{10 / 11}$ are tight or not.

Case 1: $w_{h \ell}^{01}>w_{h \ell}^{00}-L$ and $w_{h \ell}^{11}>w_{h \ell}^{10}-L$, with $\eta_{h \ell}^{00 / 01}=\eta_{h \ell}^{10 / 11}=0$.

We have $w_{h \ell}^{11} \leq w_{h \ell}^{01}$ from $N O I_{h \ell}^{01 / 11}$. If $w_{h \ell}^{11}<w_{h \ell}^{01}$, with $\delta_{h \ell}^{01 / 11}=0$, then (60) and (62) lead to a contradiction, and thus we have $w_{h \ell}^{11}=w_{h \ell}^{01}$.

We have $w_{h \ell}^{10} \leq w_{h \ell}^{00}$ from $N O I_{h \ell}^{00 / 10}$. Consider two subcases. Suppose firstly that $w_{h \ell}^{10}<w_{h \ell}^{00}$, with $\delta_{h \ell}^{00 / 10}=0$. Equations (59) and (61) then give $w_{h \ell}^{10} \geq w_{h \ell}^{01}$, and we have

$$
w_{h \ell}^{00}>w_{h \ell}^{10} \geq w_{h \ell}^{01}=w_{h \ell}^{11} .
$$

Secondly, suppose $w_{h \ell}^{10}=w_{h \ell}^{00}$. Then we have

$$
w_{h \ell}^{00}=w_{h \ell}^{10} \geq w_{h \ell}^{01}=w_{h \ell}^{11} .
$$


The same argument as in the proof of Proposition 2 shows that $w_{h \ell}^{10}=w_{h \ell}^{01}$ would contradict $u_{h h}^{*}<u_{h \ell}^{*}$. Thus we have

$$
w_{h \ell}^{00}=w_{h \ell}^{10}>w_{h \ell}^{01}=w_{h \ell}^{11},
$$

in this second subcase.

Hence, in the first subcase, an optimal insurance policy combines a deductible $D_{h \ell}=w_{h \ell}^{00}-w_{h \ell}^{10}>0$, with copays $c_{h \ell}^{1}=0, c_{h \ell}^{2} \geq 0$, and in the second one, two risk specific insurance policies are optimal, with full coverage for $r=1$ and partial coverage for $r=2$.

Case 2: $w_{h \ell}^{01}=w_{h \ell}^{00}-L$ and $w_{h \ell}^{11}=w_{h \ell}^{10}-L$, with $\eta_{h \ell}^{00 / 01} \geq 0, \eta_{h \ell}^{10 / 11} \geq 0$.

In this case, equation (2) is satisfied for type $h \ell$ households, with full or partial insurance for $r=1$ and without insurance for $r=2$.

Case 3: $w_{h \ell}^{01}=w_{h \ell}^{00}-L$ and $w_{h \ell}^{11}>w_{h \ell}^{10}-L$, with $\eta_{h \ell}^{00 / 01} \geq 0, \eta_{h \ell}^{10 / 11}=0$.

In this case, inequation (4) holds and is not binding for type $h \ell$, and thus an optimal insurance policy combines a deductible $D_{h \ell}$ with copays $c_{h \ell}^{1}$ and $c_{h \ell}^{2}$. We know from Proposition 2 that $w_{h \ell}^{10}=w_{h \ell}^{00}$ when $\sigma=0$, and thus we have $w_{h \ell}^{10}>w_{h \ell}^{00}-L$ and $c_{h \ell}^{1}<c_{h \ell}^{2}$, for $\sigma$ not too large. There is an optimal policy such that $c_{h \ell}^{1}=0, c_{h \ell}^{2}=w_{h \ell}^{10}-w_{h \ell}^{11} \in(0, L), D_{h \ell}=w_{h \ell}^{00}-w_{h \ell}^{10}>0 .{ }^{27}$

Case 4: $w_{h \ell}^{01}>w_{h \ell}^{00}-L$ and $w_{h \ell}^{11}=w_{h \ell}^{10}-L$, with $\eta_{h \ell}^{00 / 01}=0, \eta_{h \ell}^{10 / 11} \geq 0$.

Using $w_{h \ell}^{00} \geq w_{h \ell}^{10}$ from $N O I_{h \ell}^{00 / 10}$ gives $w_{h \ell}^{01}>w_{h \ell}^{11}$, and thus $\delta_{h \ell}^{01 / 11}=0$ because $N O I_{h \ell}^{01 / 11}$ is not binding. Using (60),(62) and $p_{h}>p_{\ell}$ imply $w_{h \ell}^{01} \leq$ $w_{h \ell}^{11}$, hence a contradiction. Hence, case 4 is not possible.

Combining cases 1,2 and 3 shows that an optimal insurance coverage for type $h \ell$ can be obtained either through a unique policy including a deductible $D_{h \ell}>0$ and copays $c_{h \ell}^{1}=0, c_{h \ell}^{2} \geq 0$ or through risk-specific policies with full coverage for $r=1$ and partial coverage for $r=2$ or partial coverage for $r=1$ and no coverage for $r=2$.

A symmetrical characterization is obtained for $w_{\ell h}$.

\section{3: Characterization of $w_{\ell \ell}$}

The proof of Proposition 3 may be adapted as follows. Let $w_{\ell \ell}^{n}=\left(w_{\ell \ell}^{00 n}\right.$, $\left.w_{\ell \ell}^{01 n}, w_{\ell \ell}^{10 n}, w_{\ell \ell}^{11 n}\right)$ be a sequence defined in a manner similar to the proof of

\footnotetext{
${ }^{27}$ This is similar to the case considered in footnote 9.
} 
Proposition 3. Conditions (55)-(58) show that $w_{\ell \ell}^{n}$ satisfies equations (43)(46) by replacing $\gamma$ by $\widehat{\gamma}=\gamma(1+\sigma)$ in (44)-(46) and $\gamma$ by

$$
\bar{\gamma}=\widehat{\gamma} \frac{1-(1+\sigma) p_{\ell}\left(2-p_{\ell}\right)}{\left(1-p_{\ell}\right)^{2}(1+\sigma)}<\widehat{\gamma}
$$

in (43). Let $\bar{w}_{\ell \ell}^{00 n}$ be defined by (43) with $\gamma$ replaced by $\widehat{\gamma}$ instead of $\bar{\gamma}$, and thus with $\bar{w}_{\ell \ell}^{00 n}<w_{\ell \ell}^{00 n}$. The proof of Proposition 3 shows that

$$
\bar{w}_{\ell \ell}^{00 n}+w_{\ell \ell}^{11 n}>w_{\ell \ell}^{10 n}+w_{\ell \ell}^{01 n},
$$

and thus $w_{\ell \ell}^{n}$ satifies (42) for $n$ large enough.

\section{Stage 2}

Stage 2 is the same as in the proof of Proposition 2.

\section{References}

Armstrong, M. and J.-C. Rochet (1999), "Multi-dimensional screening: A user's guide", European Economic Review, 43, 959-979.

Bourguignon, F. and P.-A. Chiappori (1992), "Collective models of household behavior", European Economic Review, 36, 355-364.

Cohen, A. (2006), "The disadvantages of aggregate deductibles", B.E. Journal of Economic Analysis and Policy, 6, Issue 1, ISSN (Online) 15380653.

Crocker, K.J. and A. Snow (1985), "The efficiency of competitive equilibria in insurance markets with asymmetric information", Journal of Public Economics, 26, 207-220.

Crocker, K.J. and A. Snow (2011), "Multidimensional screening in insurance markets with adverse selection", Journal of Risk and Insurance, 78, 287-307.

Eeckhoudt, L., L. Bauwens, E. Briys and P. Scarmure (1991), "The law of large (small?) numbers and the demand for insurance", Journal of Risk and Insurance, 58, 438-451.

Gollier, C. (2013), "The economics of optimal insurance design", in Handbook of Insurance, G. Dionne (Ed), 2nd Edition, Springer, 107-122.

Gollier, C., and H. Schlesinger (1995), "Second-best insurance contract design in an incomplete market", Scandinavian Journal of Economics, 97, 123-135. 
Li, C.-S., Liu, C.-C., and J.-H. Yeh (2007), "The incentive effects of increasing per-claim deductible contracts in automobile insurance", Journal of Risk and Insurance, 74, 441-459.

Mimra, W. and A. Wambach (2014), "New developments in the theory of adverse selection in competitive insurance", Geneva Risk and Insurance Review, 39, 136-152.

Rochet, J.-C. and L.A. Stole (2003), "The economics of multidimensional screening", in Advances in Economics and Econometrics: Theory and Applications, Eight World Congress, M. Dewatripont, L.P. Hansen, and S.J. Turnovsky (Eds), Cambridge University Press, Econometric Society Monographs.

Rothschild, M. and J.E. Stiglitz (1976), "Equilibrium in competitive insurance markets: an essay on the economics of imperfect information", Quarterly Journal of Economics, 90, 630-649.

Spence, M. (1978), "Product differentiation and performance in insurance markets", Journal of Public Economics, 10, 427-447. 